\title{
1,5-Enyne Metathesis
}

\author{
Olivier Debleds, Jean-Marc Campagne*
}

*Institut Charles Gerhardt Montpellier, Ecole Nationale Supérieur de Chimie de Montpellier, 8 Rue de l'Ecole Normale, 34296 Montpellier France

jean-marc.campagne@enscm.fr

\section{SUPPORTING INFORMATION}

\section{CONTENTS}

- General experimental details

S2

- Experimental procedures and characterization

$\mathrm{S} 3-\mathrm{S} 12$

$-{ }^{1} \mathrm{H}$ and ${ }^{13} \mathrm{C}$ NMR spectra

$\mathrm{S} 13-\mathrm{S} 36$ 


\section{General information}

Unless otherwise stated all commercial materials were used without further purification. Reactions were carried out in bottom flasks equipped with a magnetic stirring bar and sealed with a septum. Dichloromethane was distilled over $\mathrm{CaH}_{2}$. Microwaves reaction occurred in to CEM Microwaves Discover (300W). TLC analysis of all reactions was performed on silica

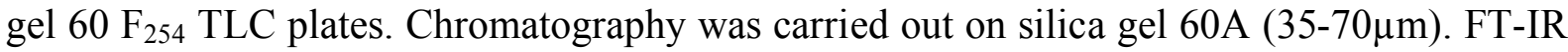
spectra were recorded with a Perkin Elem Spectrum $1000 .{ }^{1} \mathrm{H}$ and ${ }^{13} \mathrm{C}$ spectra were recorded with Bruker Ultra shield 400 plus and referenced to $\mathrm{CDCl}_{3}$ unless otherwise noted. Mass spectra and Mass spectra high resolution were obtained from the mass spectrometry operated by the Centre Commun de Spectrométrie de Masse of University Claude Bernard Lyon 1. 


\section{General procedure for cyclization of 1,5 Enyne by heating}

To a solution of 1,5 Enyne $(0.3 \mathrm{mmol})$ in $10 \mathrm{ml}$ of dichloromethane was added a first portion (5\%) of the RCM catalyst is added. The mixture was stirred and heated under reflux during 36 hours. During this period, $5 \%$ portions of the RCM catalyst are regularly added (up to $20 \%$ ) to the reaction mixture.

After reaction completion (TLC monitoring), the mixture was concentrated under vacuum and the crude material loaded on to a silica gel column and chromatographed with pentane to give the cyclization product described below.

\section{General procedure for cyclization of 1,5 Enyne by Microwaves}

To a solution of 1,5 Enyne $(0.3 \mathrm{mmol})$ in $8 \mathrm{ml}$ of dichloromethane was added the catalyst $(10 \%-20 \%$ mol.). To ensure a homogeneous system, the microwave was prestirred for $15 \mathrm{~s}$ before the irradiations were initiated. The mixture was microwave irradiated in a sealed tube for 35 minutes at $70^{\circ} \mathrm{C}$.

After 35 minutes, the mixture was concentrated under vacuum and the crude material loaded on to a silica gel column and chromatographed with pentane to give the cyclization product described below. 
Standard Reaction: Enyne (1 eq) / Hoveyda Grubbs (0,2 eq) / DCM $\min / 70^{\circ} \mathrm{C}$

Temperature (C)

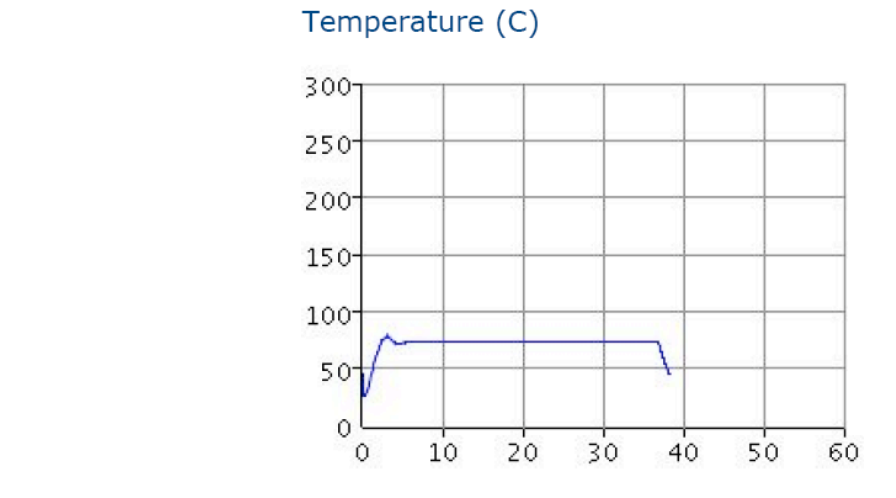

Pressure (bar)

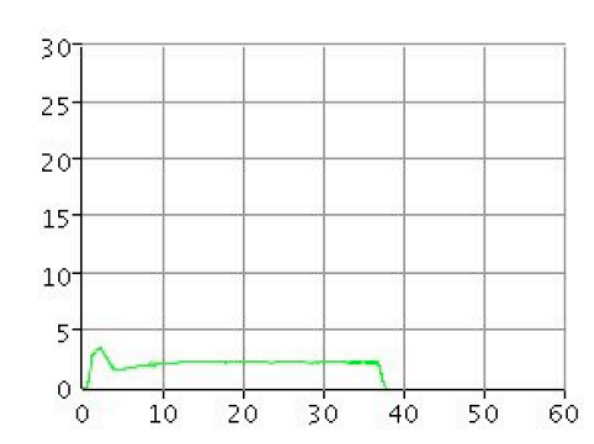

Power (W)

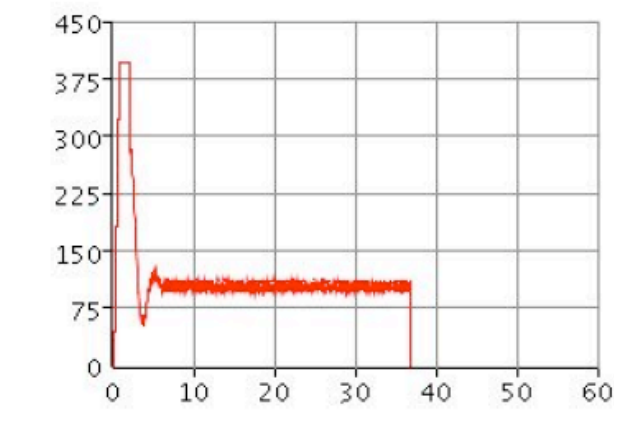

\section{min $/ 70^{\circ} \mathrm{C}$}

Micro wave : 35 
GC spectra of the crude product 2 a under standard $\boldsymbol{\mu}$-wave conditions: GC spectra crude reaction. Enyne (1 eq) / Hoveyda Grubbs (0,2 eq) / DCM Micro wave : $35 \mathrm{~min} / 70{ }^{\circ} \mathrm{C}$

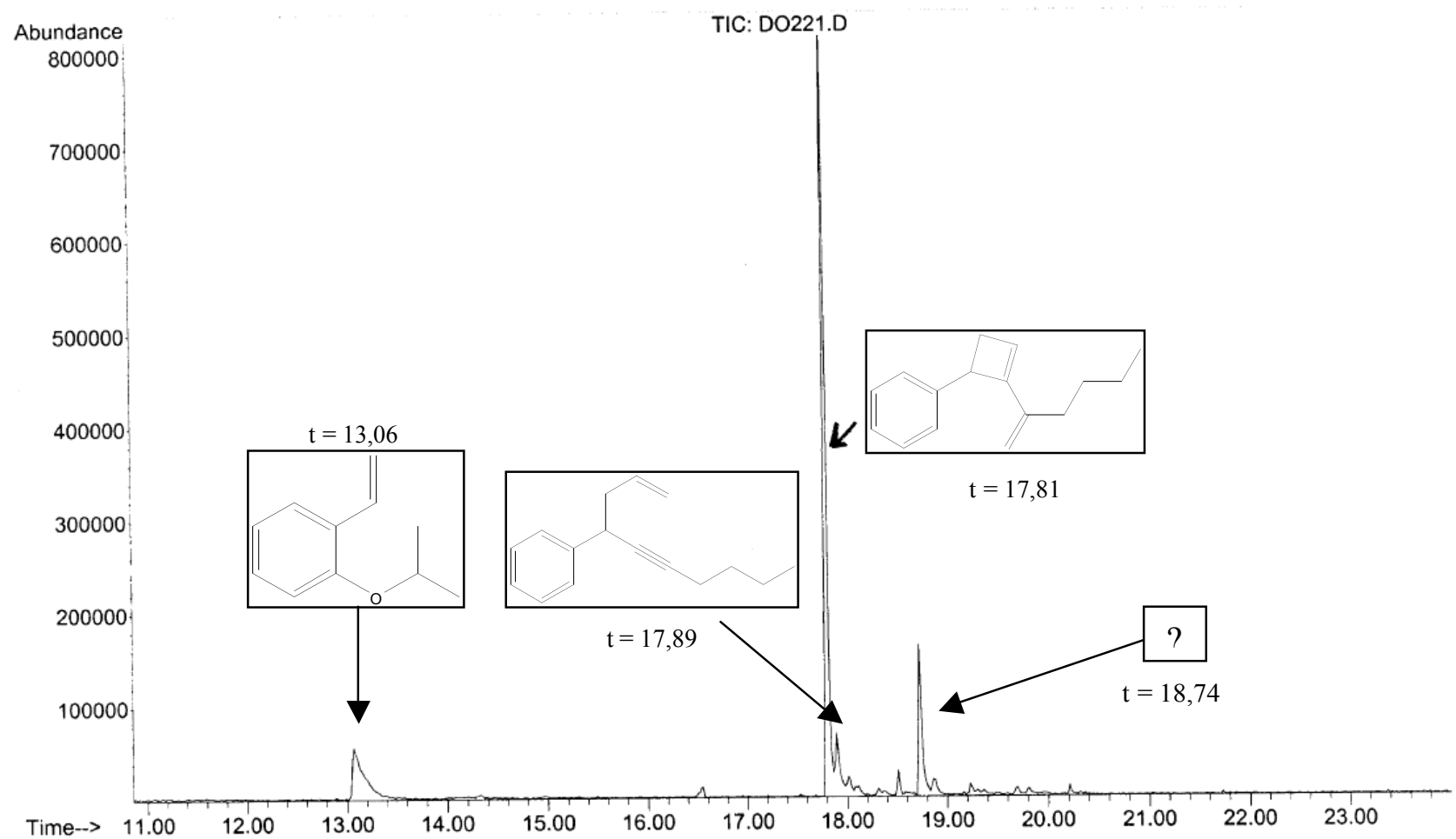

\section{GC spectra of the crude product 2a under an ethylene atmosphere (1 atm)}

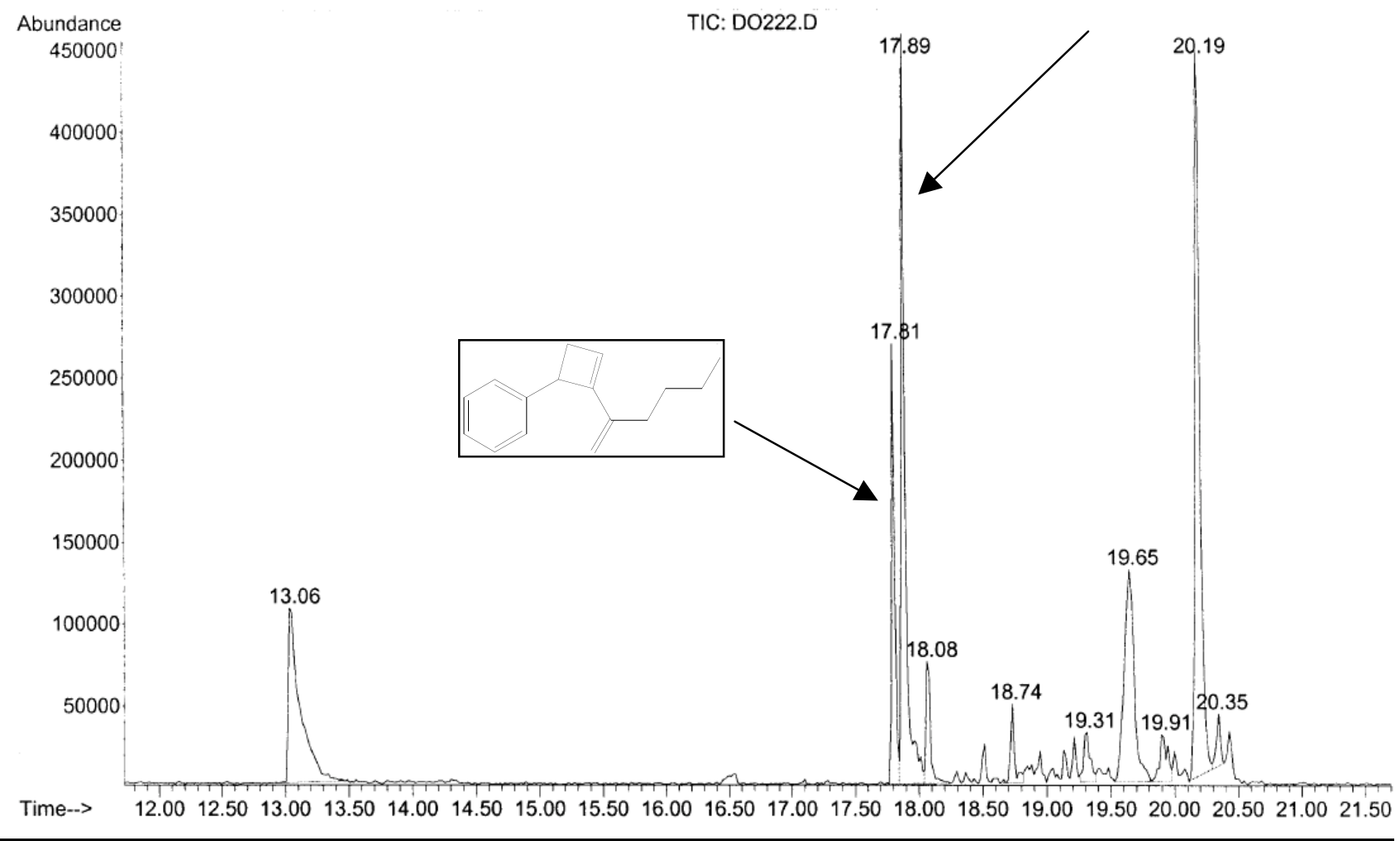


(2-(hex-1-en-2-yl) cyclobut-2-enyl) benzene (2a): Yield $58 \%$. A pale yellow oil.

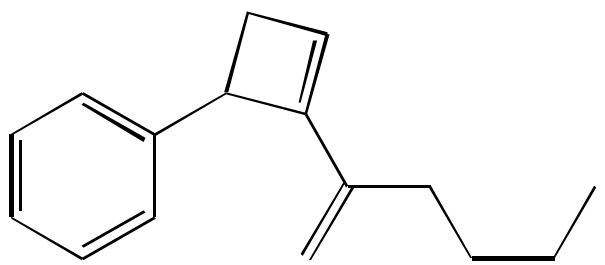

\section{${ }^{1} \mathrm{H}$ NMR (CDCl, $\left.400 \mathrm{MHz}\right)$}

$\delta 0.93(\mathrm{t}, \mathrm{J}=7.3 \mathrm{~Hz}, 3 \mathrm{H}), 1.30-1.35(\mathrm{~m}, 2 \mathrm{H}), 1.44-1.55(\mathrm{~m}, 2 \mathrm{H}), 2.21(\mathrm{~m}, 3 \mathrm{H}), 2.91(\mathrm{dd}$, $\mathrm{J}=4.8 \mathrm{~Hz}, \mathrm{~J}=14.2 \mathrm{~Hz}, 1 \mathrm{H}), 4.04(\mathrm{dd}, \mathrm{J}=1.8 \mathrm{~Hz}, \mathrm{~J}=4.8 \mathrm{~Hz}, 1 \mathrm{H}), 4.64(\mathrm{~s}, 1 \mathrm{H}), 4.77(\mathrm{~s}$, $1 \mathrm{H}), 6.15(\mathrm{~s}, 1 \mathrm{H}), 7.19-7.29(\mathrm{~m}, 5 \mathrm{H})$

\section{${ }^{13} \mathrm{C}$ NMR (CDCl $\left.3,100 \mathrm{MHz}\right)$}

$\delta 13.9,22.5,30.7,31.7,37.1,46.6,112.1,126.1,126.7$ (2C), 128.0, 128.3 (2C), 141.8, 142.9, 150.0

\section{IR-FT cm $^{-1}$}

$3025,2955,2929,2859,1601,1581,1493,1453,1377,1236,1069,1029,927,890,814,753$, 697

MS (EI)

$212(28), 187(30), 168(41), 155(100), 143(39), 131(21), 129(60), 117(42), 104(38)$, $91(52), 77(17), 55(12), 41(10)$

HRMS (EI) calc'd for $\mathrm{C}_{16} \mathrm{H}_{20}: 212.1563$, found: 212.1565

1-(2-(hex-1-en-2-yl) cyclobut-2-enyl)-2-methylbenzene (2b): Yield $52 \%$. A pale yellow oil.

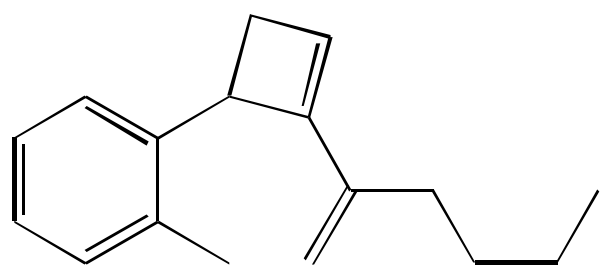

\section{${ }^{1} \mathrm{H}$ NMR ( $\left.\mathrm{CDCl}_{3}, 400 \mathrm{MHz}\right)$}

$\delta 0.96(\mathrm{t}, \mathrm{J}=7.3 \mathrm{~Hz}, 3 \mathrm{H}), 1.37-1.45(\mathrm{~m}, 2 \mathrm{H}), 1.53-1.60(\mathrm{~m}, 2 \mathrm{H}), 2.08(\mathrm{~d}, \mathrm{~J}=13.9 \mathrm{~Hz}$, $1 \mathrm{H}), 2.24-2.28(\mathrm{~m}, 2 \mathrm{H}), 2.35(\mathrm{~s}, 3 \mathrm{H}), 2.98(\mathrm{dd}, \mathrm{J}=4.9 \mathrm{~Hz}, \mathrm{~J}=13.9 \mathrm{~Hz}, 1 \mathrm{H}), 4.26(\mathrm{dd}, \mathrm{J}=$ $1.9 \mathrm{H}, \mathrm{J}=4.9 \mathrm{~Hz}, 1 \mathrm{H}), 4.67(\mathrm{~s}, 1 \mathrm{H}), 4.87(\mathrm{~s}, 1 \mathrm{H}), 6.15(\mathrm{~s}, 1 \mathrm{H}), 7.09-7.17(\mathrm{~m}, 5 \mathrm{H})$

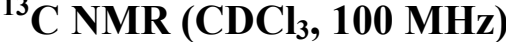

$\delta 14.0,19.2,22.6,30.8,31.7,36.2,43.0,112.3,125.8,125.8,125.9,127.7,129.6,135.6$, $140.8,142.1,149.3$

\section{IR-FT cm $^{-1}$}

$3044,2955,2939,2859,1628,1603,1583,1488,1459,1378,1236,1104,1049,928$, $889,816,797,748,723,610$

MS (EI)

$226(10), 211(6), 183(17), 169(100), 155(29), 141(38), 128(23), 115(20), 105(14)$, $91(14), 77(9), 55(7), 43(9), 41(21)$

HRMS (EI) calc'd for $\mathrm{C}_{17} \mathrm{H}_{21}: 226.1721$, found: 226.1722 
1-(2-(hex-1-en-2-yl) cyclobut-2-enyl)-3-methylbenzene (2c):Yield $47 \%$. A pale yellow oil.

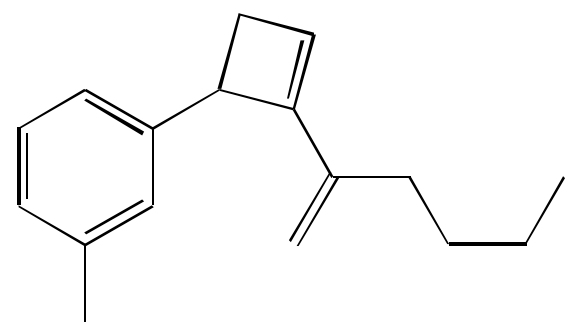

\section{${ }^{1} \mathrm{H}$ NMR $\left(\mathrm{CDCl}_{3}, 400 \mathrm{MHz}\right)$}

$\delta 0.93(\mathrm{t}, \mathrm{J}=7.3 \mathrm{~Hz}, 3 \mathrm{H}), 1.26-1.35(\mathrm{~m}, 2 \mathrm{H}), 1.47-1.56(\mathrm{~m}, 2 \mathrm{H}), 2.19(\mathrm{~m}, 3 \mathrm{H}), 2.32(\mathrm{~s}$, $3 \mathrm{H}), 2.89$ (ddd, $\mathrm{J}=0.95 \mathrm{~Hz}, \mathrm{~J}=4.8 \mathrm{~Hz}, \mathrm{~J}=14.1 \mathrm{~Hz}, 1 \mathrm{H}), 4.00(\mathrm{dd}, \mathrm{J}=1.8 \mathrm{~Hz}, \mathrm{~J}=4.8 \mathrm{~Hz}$, $1 \mathrm{H}), 4.65(\mathrm{~s}, 1 \mathrm{H}), 4.77(\mathrm{~s}, 1 \mathrm{H}), 6.14(\mathrm{~s}, 1 \mathrm{H}), 6.99-7.03(\mathrm{~m}, 3 \mathrm{H}), 7.18(\mathrm{t}, \mathrm{J}=7.6 \mathrm{~Hz}, 1 \mathrm{H})$

${ }^{13} \mathrm{C}$ NMR (CDCl $\left.3,100 \mathrm{MHz}\right)$

$\delta 13.9,21.4,22.5,30.7,31.7,37.1,46.5,112.1,123.7,126.9,127.4,127.9,128.1,137.7$, $141.8,142.8,150.1$

IR-FT cm $^{-1}$

$3033,2931,2870,1719,1676,1606,1576,1488,1456,1384,1325,1236,1039,930$, $889,809,779,700$

MS(EI)

$226(20), 211(4), 183(11), 169(100), 154(34), 141(30), 128(23), 115(18), 105(15)$, $91(14), 77(11), 55(7), 43(10), 41(23)$

HRMS (EI) calc'd for $\mathrm{C}_{17} \mathrm{H}_{21}: 226.1721$, found: 226.1722

1-chloro-4-(2-(hex-1-en-2-yl) cyclobut-2-enyl) benzene (2d): Yield: 37 \%. A pale yellow oil<smiles>C=C(CCCC)C1=CCC1c1ccc(Cl)cc1</smiles>

\section{${ }^{1} \mathrm{H}$ NMR ( $\left.\mathrm{CDCl}_{3}, 400 \mathrm{MHz}\right)$}

$\delta 0.92(\mathrm{t}, \mathrm{J}=7.3 \mathrm{~Hz}, 3 \mathrm{H}), 1.29-1.48(\mathrm{~m}, 4 \mathrm{H}), 2.18(\mathrm{~m}, 3 \mathrm{H}), 2.91(\mathrm{ddd}, \mathrm{J}=0.8, \mathrm{~J}=4.8 \mathrm{~Hz}, \mathrm{~J}$ $=14.1 \mathrm{~Hz}, 1 \mathrm{H}), 4.00(\mathrm{dd}, \mathrm{J}=1.6 \mathrm{~Hz}, \mathrm{~J}=4.8 \mathrm{~Hz}, 1 \mathrm{H}), 4.58(\mathrm{~s}, 1 \mathrm{H}), 4.77(\mathrm{~s}, 1 \mathrm{H}), 6.14(\mathrm{~s}$, $1 \mathrm{H}), 7.2(\mathrm{~m}, 2 \mathrm{H}), 7.25(\mathrm{~m}, 2 \mathrm{H})$

${ }^{13} \mathrm{C}$ NMR (CDCl, $\left.100 \mathrm{MHz}\right)$

$\delta 13.9,22.5,30.7,31.6,37.0,45.8,112.2,128.0(2 \mathrm{C}), 128.2,128.4(2 \mathrm{C}), 131.6,141.4,142.7$, 149.7

IR-FT cm $^{-1}$

$3033,2956,2930,2871,1738,1676,1576,1490,1465,1407,1384,1236,1091$, 1014,823

MS (EI)

$246(20), 231(4), 203(11), 189(100), 174(34), 161(30), 148(23), 139(8), 115(18)$, 105 (15), $91(14), 77(11), 55(7), 43(10), 41(23)$

HRMS (EI) calc'd for $\mathrm{C}_{16} \mathrm{H}_{19} \mathrm{Cl}$ : 246.1175, found: 246.1175 
1-fluoro-4-(2-(hex-1-en-2-yl) cyclobut-2-enyl) benzene (2e): Yield: $35 \%$. A pale yellow oil<smiles>C=C(CCCC)C1=CCC1c1ccc(F)cc1</smiles>

\section{${ }^{1} \mathrm{H}$ NMR $\left(\mathrm{CDCl}_{3}, 400 \mathrm{MHz}\right)$}

$\delta 0.92(\mathrm{t}, \mathrm{J}=7.3 \mathrm{~Hz}, 3 \mathrm{H}), 1.29-1.38(\mathrm{~m}, 2 \mathrm{H}, 1.45), 1.53(\mathrm{~m}, 2 \mathrm{H}), 2.18(\mathrm{~m}, 3 \mathrm{H}), 2.9$ (ddd, J $=0.9, \mathrm{~J}=4.8 \mathrm{~Hz}, \mathrm{~J}=14.1 \mathrm{~Hz}, 1 \mathrm{H}), 4.01(\mathrm{dd}, \mathrm{J}=1.8 \mathrm{~Hz}, \mathrm{~J}=4.8 \mathrm{~Hz}, 1 \mathrm{H}), 4.60(\mathrm{~s}, 1 \mathrm{H}), 4.77$ $(\mathrm{s}, 1 \mathrm{H}), 6.13(\mathrm{~s}, 1 \mathrm{H}), 6.96(\mathrm{~m}, 2 \mathrm{H}), 7.15(\mathrm{~m}, 2 \mathrm{H})$

\section{${ }^{13} \mathrm{C}$ NMR (CDCl $\left.3,100 \mathrm{MHz}\right)$}

$\delta 13.9,22.3,30.7,31.6,37.2,45.8,112.1,114.9(\mathrm{~d}, 2 \mathrm{C}), 128.0(\mathrm{~d}, 2 \mathrm{C}), 128.1,138.5,141.7$, $149.9,160.8(\mathrm{~d})$

\section{IR-FT cm $^{-1}$}

$2957,2929,2871,1676,1603,1508,1465,1260,1223,1157,1094,1015,831,795$

MS (EI)

230 (18), 201 (9) , $187(26), 173(100), 159$ (38), 153 (22), $146(27), 133(21), 122(12)$, $109(47), 101(5), 91(6), 79(9), 41(8)$

HRMS (EI) calc'd for $\mathrm{C}_{16} \mathrm{H}_{19} \mathrm{~F}: 230.1470$, found: 230.1471

4-(2-(hex-1-en-2-yl) cyclobut-2-enyl) biphenyl (2f): Yield: $57 \%$. A pale yellow oil.<smiles>C=C(CCCC)C1=CCC1c1ccc(-c2ccccc2)cc1</smiles>

${ }^{1} \mathrm{H}$ NMR $\left(\mathrm{CDCl}_{3}, 400 \mathrm{MHz}\right) \delta \mathrm{ppm}$

$\delta 0.92(\mathrm{t}, \mathrm{J}=7.3 \mathrm{~Hz}, 3 \mathrm{H}), 1.31-1.41(\mathrm{~m}, 2 \mathrm{H}) 1.48-1.57(\mathrm{~m}, 2 \mathrm{H}), 2.23(\mathrm{~m}, 3 \mathrm{H}), 2.95(\mathrm{ddd}$, $\mathrm{J}=0.7, \mathrm{~J}=4.8 \mathrm{~Hz}, \mathrm{~J}=14.1 \mathrm{~Hz}, 1 \mathrm{H}), 4.09(\mathrm{dd}, \mathrm{J}=1.7 \mathrm{~Hz}, \mathrm{~J}=4.8 \mathrm{~Hz}, 1 \mathrm{H}), 4.69(\mathrm{~s}, 1 \mathrm{H}), 4.80$ $(\mathrm{s}, 1 \mathrm{H}), 6.18(\mathrm{~s}, 1 \mathrm{H}), 7.29-7.34(\mathrm{~m}, 3 \mathrm{H}), 7.4-7.44(\mathrm{~m}, 2 \mathrm{H}), 7.51-7.53(\mathrm{~m}, 2 \mathrm{H}), 7.58-$ 7.60 (m, 2H)

${ }^{13} \mathrm{C}$ NMR $\left(\mathrm{CDCl}_{3}, 100 \mathrm{MHz}\right)$

$\delta$ 14.0, 22.5, 30.7, 31.7, 37.1, 45.2, 112.2, 126.9, 127.0 (2C), $127.1(2 \mathrm{C}), 127.2(2 \mathrm{C}), 128.1$, $128.6(2 \mathrm{C}), 139.0,141.1,141.8,142.1,150.0$

IR-FT cm$^{-1}$

$3028,2955,2929,2870,1676,1600,1581,1486,1465,1408,1378,1236,1075$, $1007,892,832,763,730,697,570,505$

MS (EI)

$288(30), 245(28), 231(100), 215(32), 202(18), 189(6), 178(10), 167(26), 152(14)$, $128(4), 115(9), 91(10), 77(5), 41(4)$ 
HRMS (EI) calc' $d$ for $\mathrm{C}_{22} \mathrm{H}_{24}: 288.1877$, found: 288.1878

1-(2-(hex-1-en-2-yl) cyclobut-2-enyl)-4-methoxybenzene (2g): Yield: 53 \%. A pale yellow oil.

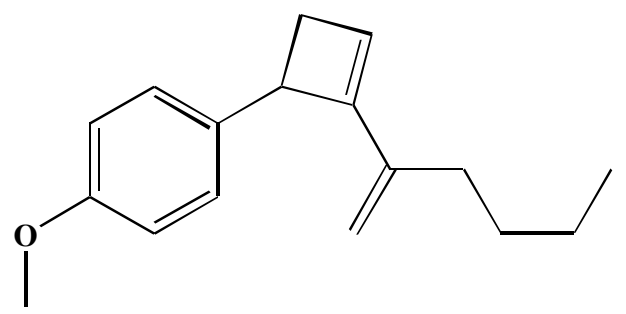

\section{${ }^{1} \mathrm{H}$ NMR $\left(\mathrm{CDCl}_{3}, 400 \mathrm{MHz}\right)$}

$\delta 0.93(\mathrm{t}, \mathrm{J}=7.3 \mathrm{~Hz}, 3 \mathrm{H}), 1.26-1.36(\mathrm{~m}, 2 \mathrm{H}), 1.45-1.53(\mathrm{~m}, 2 \mathrm{H}), 2.19(\mathrm{~m}, 3 \mathrm{H}), 2.89$ (ddd, J = 0.7, J = 4.8 Hz, J =14.0 Hz, 1H), $3.78(\mathrm{~s}, 3 \mathrm{H}), 3.99(\mathrm{dd}, \mathrm{J}=1,7 \mathrm{~Hz}, \mathrm{~J}=4.8 \mathrm{~Hz}$, $1 \mathrm{H}), 4.64(\mathrm{~s}, 1 \mathrm{H}), 4.77(\mathrm{~s}, 1 \mathrm{H}), 6.13(\mathrm{~s}, 1 \mathrm{H}), 6.8(\mathrm{~m}, 2 \mathrm{H}), 7.14(\mathrm{~m}, 2 \mathrm{H})$

${ }^{13} \mathrm{C}$ NMR $\left(\mathrm{CDCl}_{3}, 100 \mathrm{MHz}\right) \delta \mathrm{ppm}$

$\delta 13.9,22.5,30.7,31.7,37.3,45.8,55.1,112.0,113.7$ (2C), $127.6(2 \mathrm{C}), 127.9,135.0,141.8$, $150.2,157.9$

\section{IR-FT cm $^{-1}$}

$3024,2929,2858,2834,1733,1610,1583,1510,1464,1301,1243,1174,1107$, $1037,956,926,890,826,752,691$

MS (EI)

$242(100), 227$ (7) , $213(32), 200(38), 191(42), 185(74), 171(45), 155(17), 141(31)$, $121(61), 115(22), 77(28), 55(12), 41(17)$

HRMS (EI) calc'd for $\mathrm{C}_{17} \mathrm{H}_{22} \mathrm{O}: 242.1672$, found: 242.1671

4-(2-(hex-1-en-2-yl) cyclobut-2-enyl) phenol (2h): Yield $48 \%$. A pale yellow oil.<smiles>C=C(CCCC)C1=CCC1c1ccc(O)cc1</smiles>

\section{${ }^{1} \mathrm{H}$ NMR $\left(\mathrm{CDCl}_{3}, 400 \mathrm{MHz}\right)$}

$\delta 0.92(\mathrm{t}, \mathrm{J}=7.3 \mathrm{~Hz}, 3 \mathrm{H}), 1.28-1.36(\mathrm{~m}, 2 \mathrm{H}), 1.45-1.53(\mathrm{~m}, 2 \mathrm{H}), 2.17(\mathrm{~m}, 3 \mathrm{H}), 2.71$ (ddd, $\mathrm{J}=0.7, \mathrm{~J}=4,8 \mathrm{~Hz}, \mathrm{~J}=14.0 \mathrm{~Hz}, 1 \mathrm{H}), 3.97(\mathrm{dd}, \mathrm{J}=1.7 \mathrm{~Hz}, \mathrm{~J}=4.8 \mathrm{~Hz} .1 \mathrm{H}), 4.64(\mathrm{~s}$, $1 \mathrm{H}), 4.77(\mathrm{~s}, 1 \mathrm{H}), 4.95(\mathrm{~s}, 1 \mathrm{H}), 6.12(\mathrm{~s}, 1 \mathrm{H}), 6.76(\mathrm{~d}, \mathrm{~J}=8.51 \mathrm{~Hz}, 2 \mathrm{H}), 7.09(\mathrm{~d}, \mathrm{~J}=8.51$ $\mathrm{Hz}, 2 \mathrm{H})$

${ }^{13} \mathrm{C}$ NMR $\left(\mathrm{CDCl}_{3}, 100 \mathrm{MHz}\right)$

$\delta 13.7,22.5,30.7,31.7,37.3,45.8,112.0,115.1(2 \mathrm{C}), 127.8(2 \mathrm{C}), 127.9,135.1,141.8,150.1$, 153.7

\section{IR-FT cm $^{-1}$}

$3284,3033,2912,2863,1609,1508,1439,1373,1215,1170,1096,1063$, 1015,923 , $886,821,698,687,647,539$

MS (EI)

$229(100), 215(4), 187(2), 171(3), 135(8), 133(6), 107(9), 93(2), 81(2), 71(2)$

HRMS (EI) calc'd for $\mathrm{C}_{16} \mathrm{H}_{20} \mathrm{O}: 229.1593$, found: 229.1592 
(2-(4-phenylbut-1-en-2-yl) cyclobut-2-enyl) benzene (2i): Yield: $39 \%$. A pale yellow oil.

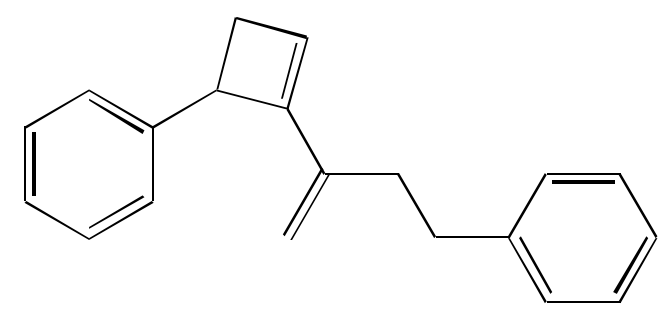

\section{${ }^{1} \mathrm{H}$ NMR $\left(\mathrm{CDCl}_{3}, 400 \mathrm{MHz}\right)$}

$\delta 2.22(\mathrm{~d}, \mathrm{~J}=14.3 \mathrm{~Hz}, 1 \mathrm{H}), 2.51(\mathrm{~m}, 2 \mathrm{H}), 2.83(\mathrm{~m}, 2 \mathrm{H}), 2.93(\mathrm{ddd}, \mathrm{J}=0.95 \mathrm{~Hz}, \mathrm{~J}=4.84 \mathrm{~Hz}$, $\mathrm{J}=14.18 \mathrm{~Hz}, 1 \mathrm{H}), 4.05(\mathrm{dd}, \mathrm{J}=., 81 \mathrm{~Hz}, \mathrm{~J}=4.84 \mathrm{~Hz}, 1 \mathrm{H}), 4.68(\mathrm{~s}, 1 \mathrm{H}), 4.80(\mathrm{~s}, 1 \mathrm{H}), 6.20$ $(\mathrm{s}, 1 \mathrm{H}), 7.18-7.32(\mathrm{~m}, 10 \mathrm{H})$

\section{${ }^{13} \mathrm{C} \mathrm{NMR}\left(\mathrm{CDCl}_{3}, 100 \mathrm{MHz}\right)$}

$\delta 33.9,35.0,37.2,46.6,112.7,125.8,126.2,126.7$ (2C), 128.3 (2C), $128.3(3 \mathrm{C}), 128.4(2 \mathrm{C})$, $141.0,142.1,142.7,149.8$

\section{IR-FT cm $^{-1}$}

$3084,3061,3026,2916,2856,1724,1675,1602,1582,1494,1453,1238,1194,1070$ , 1030, 925, 894, 816

MS (EI)

$260(6), 245(5), 232(4), 219(7), 199$ (9) , $185(13), 169(16), 159(18), 155(21), 141$ (17) , $129(32), 111(14), 105(25), 97(14), 91(100), 85(13), 71(12), 51(12), 49(12)$

HRMS (EI) calc'd for $\mathrm{C}_{20} \mathrm{H}_{20} \mathrm{O}: 260.1565$, found: 260.1562

(1-(4-phenylcyclobut-1-enyl) vinyl) benzene (2k): Yield: $2 \%$. A pale yellow oil.<smiles>C=C(C1=CCC1c1ccccc1)c1ccccc1</smiles>

\section{${ }^{1} \mathrm{H}$ NMR $\left(\mathrm{CDCl}_{3}, 400 \mathrm{MHz}\right)$}

$\delta 2.27(\mathrm{~d}, \mathrm{~J}=14.5 \mathrm{~Hz}, 1 \mathrm{H}), 2.97(\mathrm{ddd}, \mathrm{J}=1.0, \mathrm{~J}=4.9 \mathrm{~Hz}, \mathrm{~J}=14.4 \mathrm{~Hz}, 1 \mathrm{H}), 4.17(\mathrm{dd}, \mathrm{J}=1.8$ $\mathrm{Hz}, \mathrm{J}=4.9 \mathrm{~Hz}, 1 \mathrm{H}), 4.91(\mathrm{~s}, 1 \mathrm{H}), 5.09(\mathrm{~s}, 1 \mathrm{H}), 6.15(\mathrm{~s}, 1 \mathrm{H}), 7.2-7.45(\mathrm{~m}, 10 \mathrm{H})$

${ }^{13} \mathrm{C}$ NMR $\left(\mathrm{CDCl}_{3}, \mathbf{1 0 0} \mathrm{MHz}\right)$

$\delta 37.3,46.7,114.8,126.3,126.7,127.5$ (2C), 127.7 (2C), 128.2 (2C), $128.4(2 \mathrm{C}), 131.3$, $139.2,142.5,148.2,168.2$

Due to the low yield obtained in this reaction and instability of the cyclobutene, MS and HRMS analysis could not be carried out. 
1-(hex-1-en-2-yl)-4,4-dimethylcyclobut-1-ene (2I): Yield: $38 \%$. A pale yellow oil.

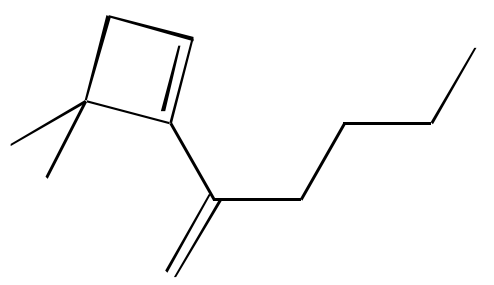

\section{${ }^{1} \mathrm{H}$ NMR $\left(\mathrm{CDCl}_{3}, 400 \mathrm{MHz}\right)$}

$\delta 0.91(\mathrm{t}, \mathrm{J}=7.2 \mathrm{~Hz}, 3 \mathrm{H}), 1.27(\mathrm{~s}, 6 \mathrm{H}), 1.24-1.37(\mathrm{~m}, 2 \mathrm{H}), 1.42-1.49(\mathrm{~m}, 2 \mathrm{H}), 2.15(\mathrm{~m}$, $4 \mathrm{H}), 4.78(\mathrm{~s}, 1 \mathrm{H}), 4.80(\mathrm{~s}, 1 \mathrm{H}), 5.76(\mathrm{~s}, 1 \mathrm{H})$

${ }^{13} \mathrm{C}$ NMR (CDCl $\left.3,100 \mathrm{MHz}\right)$

$\delta 14.0,22.5,25.5(2 \mathrm{C}), 30.8,32.4,42.8,43.9,110.6,124.0,141.9,155.4$

IR-FT cm $^{-1}$

$2959,2926,2856,1742,1580,1460,1378,1362,1248,1024,937,885,798,728$

Cyclobutene is highly unstable; MS and HRMS could not be carried out

1,4-bis (2-(hex-1-en-2-yl) cyclobut-2-enyl) benzene (4): Yield: 19\%. A pale yellow oil.

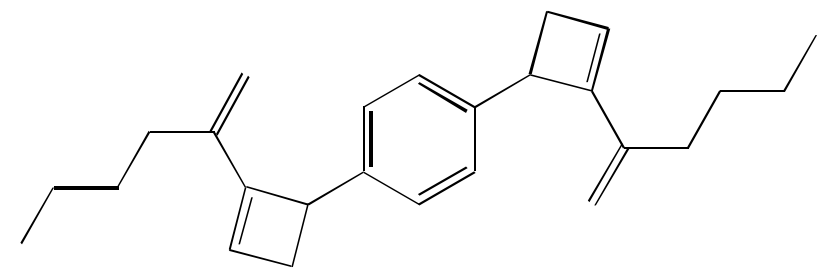

\section{${ }^{1} \mathrm{H}$ NMR $\left(\mathrm{CDCl}_{3}, 400 \mathrm{MHz}\right)$}

$\delta 0.91(\mathrm{t}, \mathrm{J}=7.3 \mathrm{~Hz}, 6 \mathrm{H}), 1.28-1,36(\mathrm{~m}, 4 \mathrm{H}), 1.45-1.54(\mathrm{~m}, 4 \mathrm{H}), 2.19(\mathrm{~m}, 6 \mathrm{H}), 2.85(\mathrm{dd}$, $\mathrm{J}=4.8 \mathrm{~Hz}, \mathrm{~J}=14.2 \mathrm{~Hz}, 2 \mathrm{H}), 4.00(\mathrm{dd}, \mathrm{J}=1.6 \mathrm{~Hz}, \mathrm{~J}=4.8 \mathrm{~Hz}, 2 \mathrm{H}), 4.65(\mathrm{~s}, 2 \mathrm{H}), 4.76(\mathrm{~s}$, $2 \mathrm{H}), 6.12(\mathrm{~s}, 2 \mathrm{H}), 7.12(\mathrm{~s}, 4 \mathrm{H})$

${ }^{13} \mathrm{C}$ NMR ( $\left.\mathrm{CDCl}_{3}, 100 \mathrm{MHz}\right)$

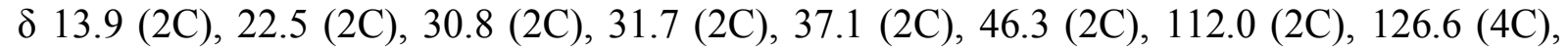
127.9 (2C), $141.8(2 \mathrm{C}), 142.8(2 \mathrm{C}), 150.1,150.2$

\section{IR-FT cm$^{-1}$}

$3082,3044,2956,2927,2858,1681,1631,1583,1509,1465,1378,1236,1192$, $1107,1075,1020,959,927,890,809$

MS (EI)

$346(13), 303$ (17) , $289(58), 211(100), 205(18), 195(14), 181(66), 167$ (46) , $155(31)$, $141(28), 129(19), 117(18), 91(13), 55(18), 41(15)$

HRMS (EI) calc'd for $\mathrm{C}_{26} \mathrm{H}_{34}: 346.2662$, found: 346.2661 


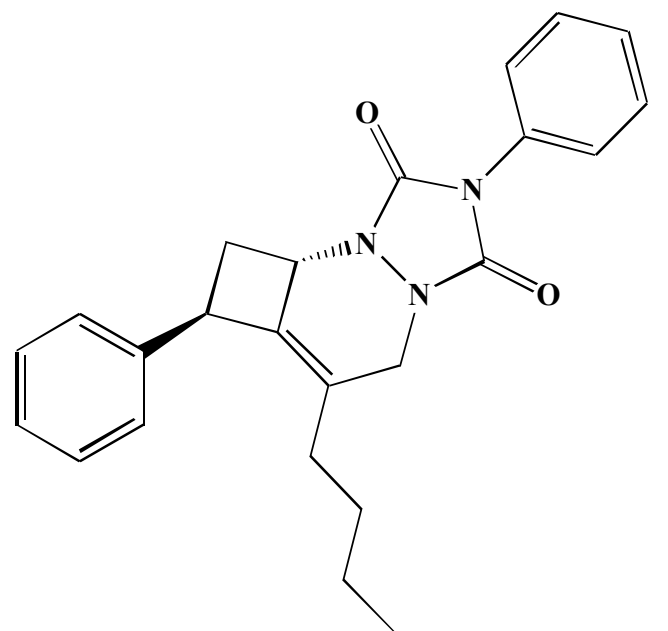

Yield 80\%. A pale yellow oil.

\section{${ }^{1} \mathrm{H}$ NMR (Acetone, $\left.400 \mathrm{MHz}\right)$}

$\delta 0.90(\mathrm{t}, \mathrm{J}=7.3 \mathrm{~Hz}, 3 \mathrm{H}), 1.33-1.42(\mathrm{~m}, 2 \mathrm{H}), 1.47-1.61(\mathrm{~m}, 2 \mathrm{H}), 2.21(\mathrm{~m}, 1 \mathrm{H}), 2.39(\mathrm{~m}$, $1 \mathrm{H}), 2.55(\mathrm{ddd}, \mathrm{J}=2.5 \mathrm{~Hz}, \mathrm{~J}=7,7 \mathrm{~Hz}, \mathrm{~J}=10.9 \mathrm{~Hz}, 1 \mathrm{H}), 2.72(\mathrm{ddd}, \mathrm{J}=7.7 \mathrm{~Hz}, 8.9 \mathrm{~Hz}, 10.9$ $\mathrm{Hz}, 1 \mathrm{H}), 4.04(\mathrm{~d}, \mathrm{~J}=16.1 \mathrm{~Hz}, 1 \mathrm{H}), 4.44(\mathrm{~d}, \mathrm{~J}=16.1 \mathrm{~Hz}, 1 \mathrm{H}), 4.53(\mathrm{~d}, \mathrm{~J}=8.9 \mathrm{~Hz}, 1 \mathrm{H}), 4.77$ (dddd, $\mathrm{J}=1.1 \mathrm{~Hz}, \mathrm{~J}=2.4 \mathrm{~Hz}, \mathrm{~J}=5.1 \mathrm{~Hz}$ et $\mathrm{J}=8.7 \mathrm{~Hz}, 1 \mathrm{H}), 7.24-7.56(\mathrm{~m}, 10 \mathrm{H})$

\section{${ }^{13} \mathrm{C} \mathrm{NMR}\left(\mathrm{CDCl}_{3}, 100 \mathrm{Mhz}\right)$}

$\delta$ 13.9, 22.4, 29.1, 29.9, 34.8, 45.2, 47.1, 55.5, 124.8, 125.4 (2C), 126.7, 127.0 (2C), 128.0, 128.8 (2C), 129.1 (2C), 129.31, 131.2, 141.1, 151.0, 153.3

\section{IR-FT cm $^{-1}$}

$3062,3026,2956,2930,2871,1948,1870,1774,1715,1600,1503,1456,1417$, $1341,1291,1240,1133,1072,1028,995,910,821,765,734,699,643,567,505$

Masse (EI)

387 (26) , $296(100), 177(38), 155$ (4) , 128 (3), 91 (10), 77 (2)

HRMS (EI) calc'd for $\mathrm{C}_{24} \mathrm{H}_{25} \mathrm{~N}_{3} \mathrm{O}_{2}: 387.1946$, found: 387.1947

\section{NOESY effects observed:}

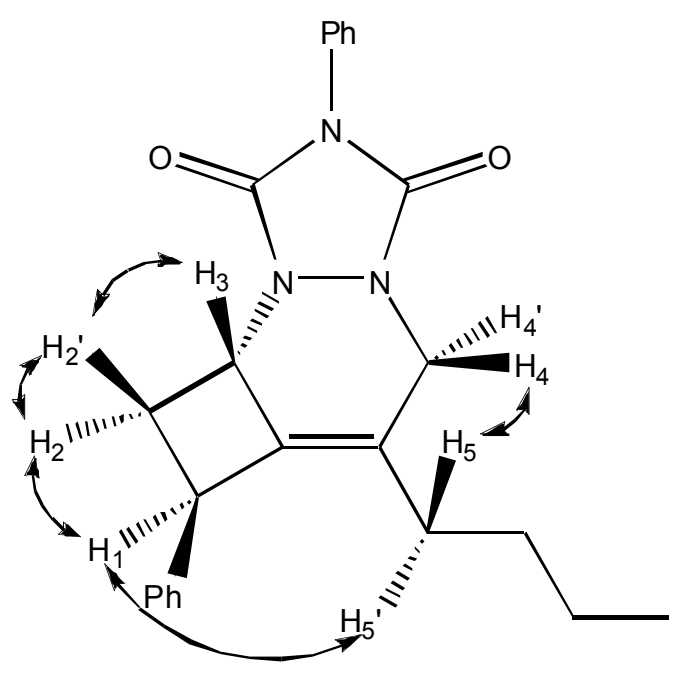




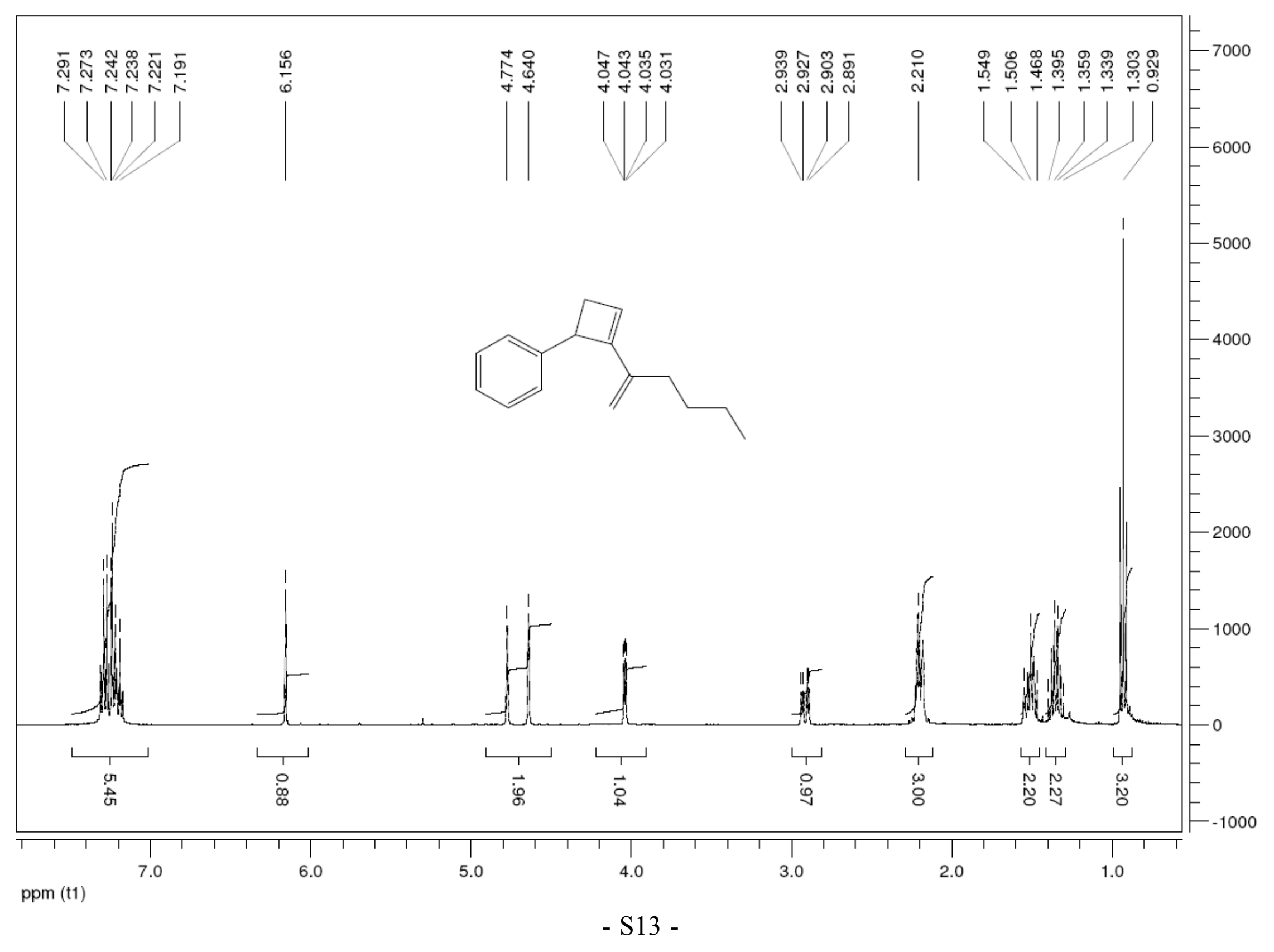




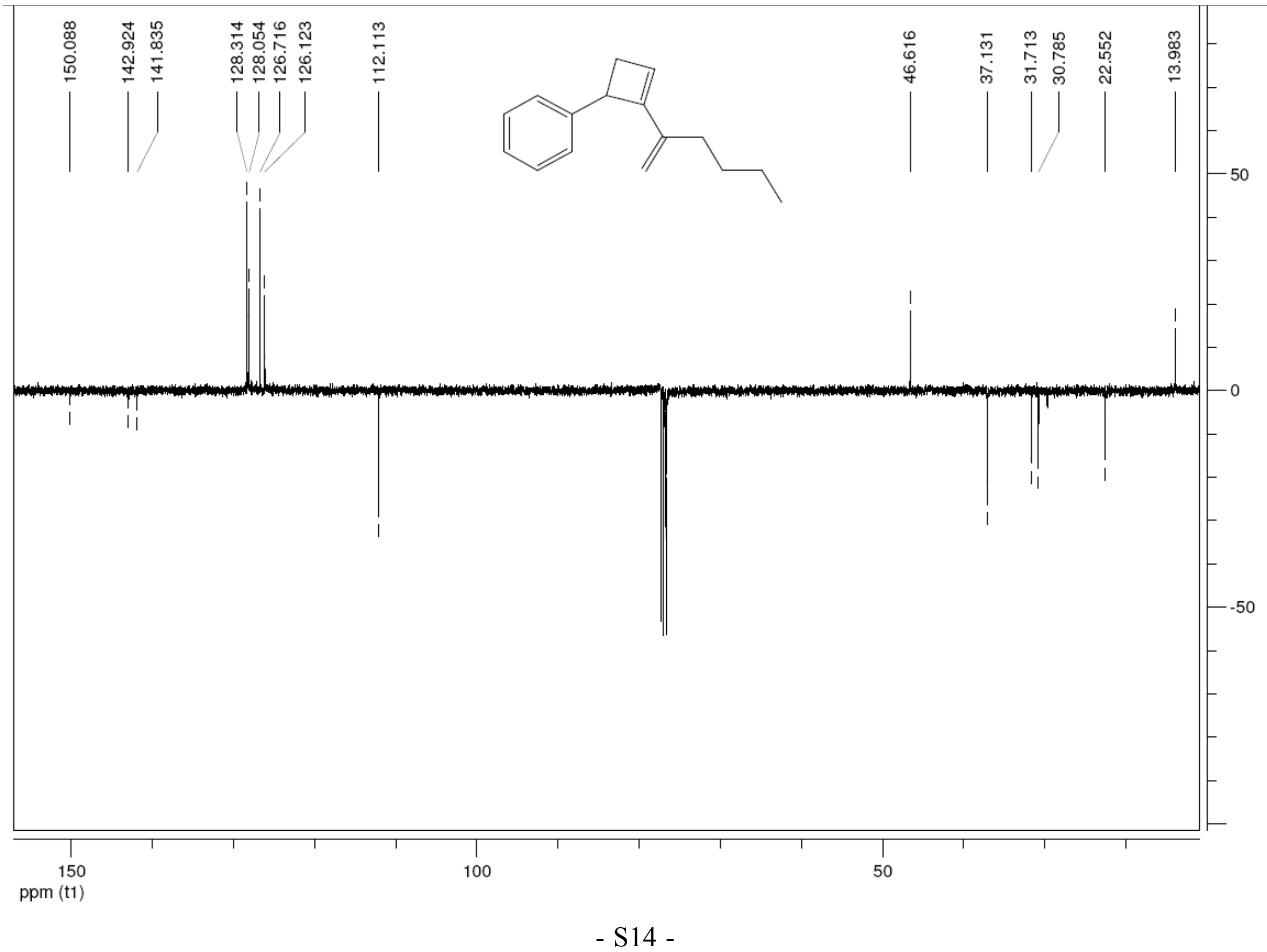




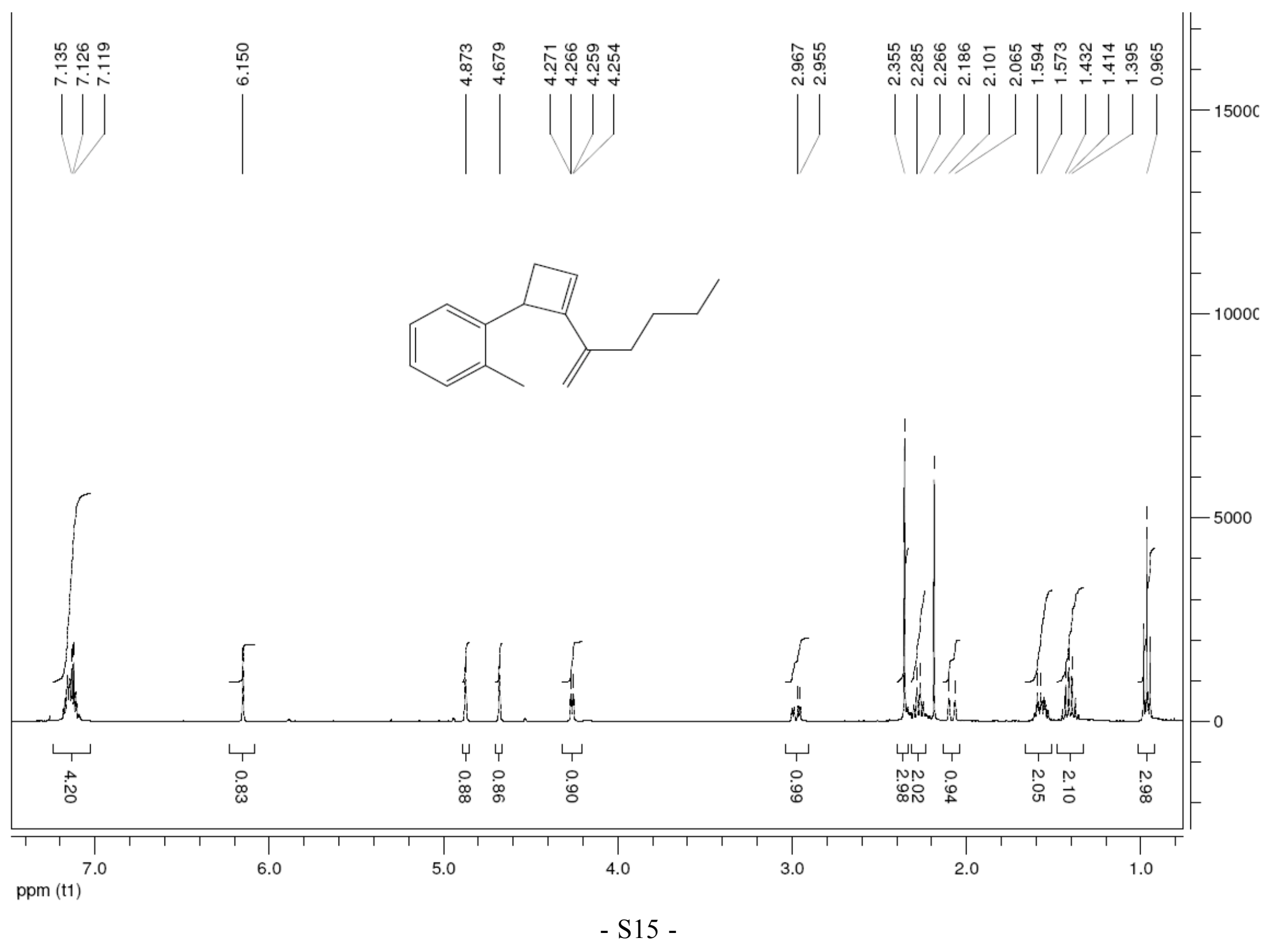




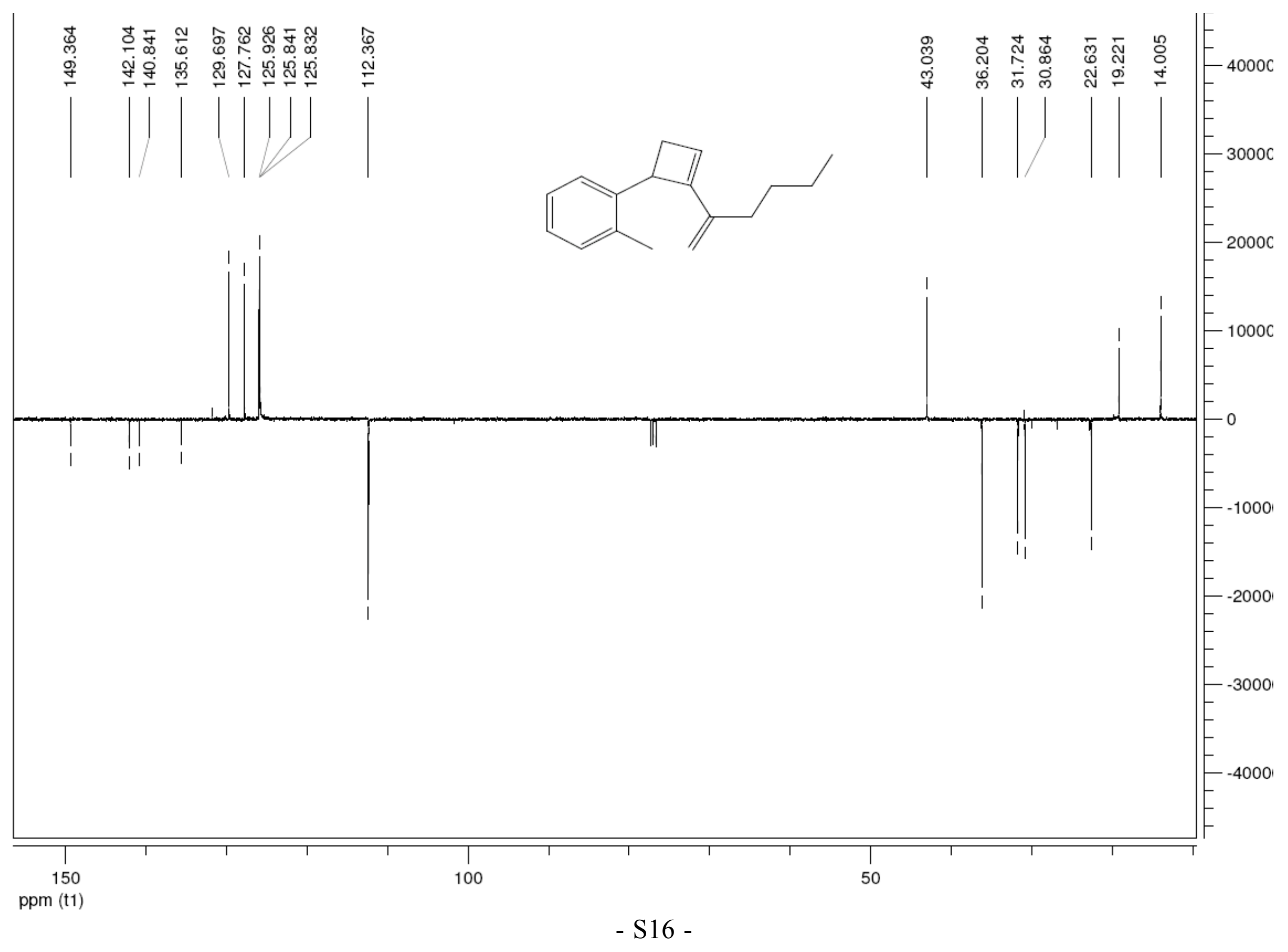




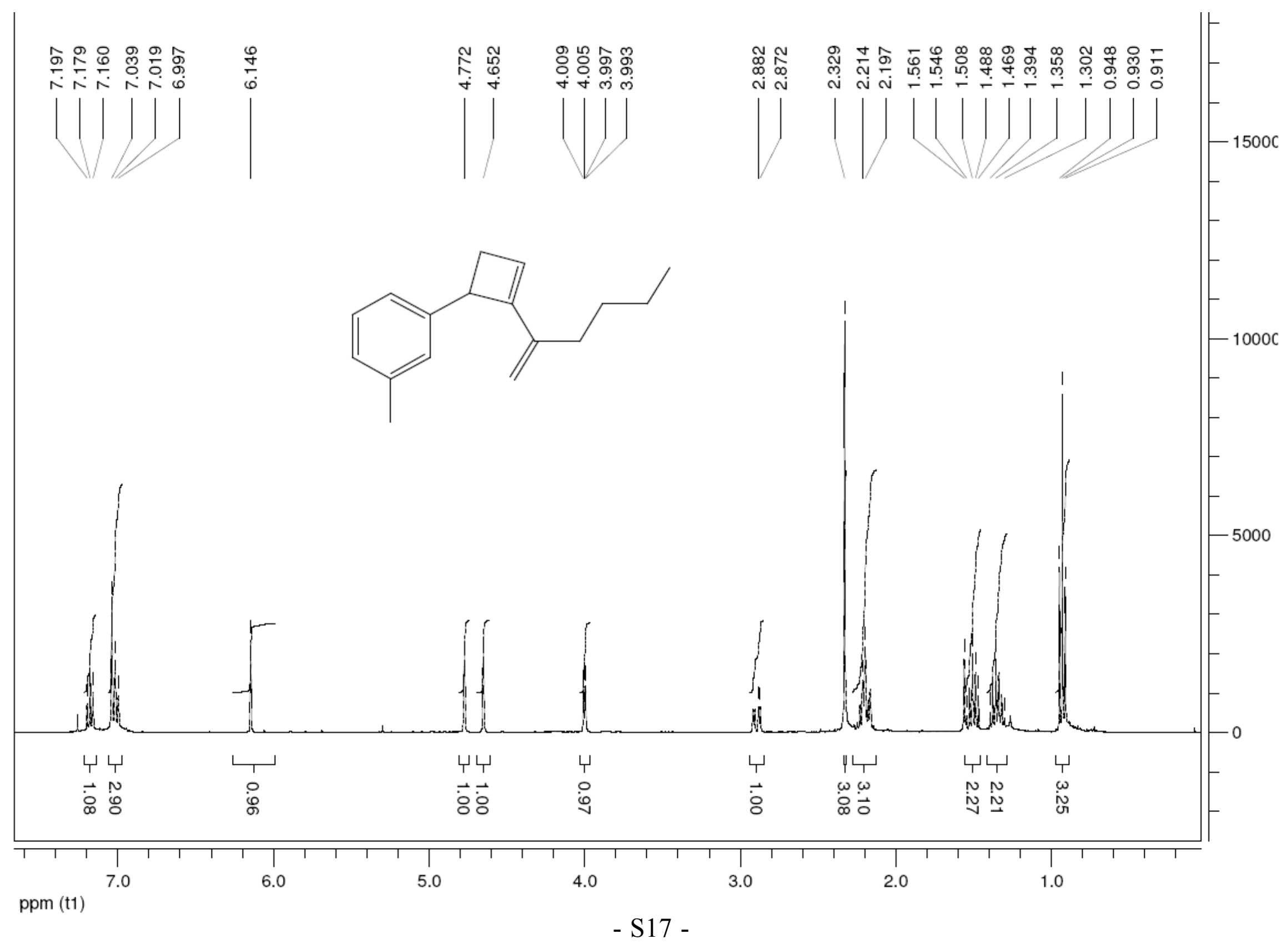




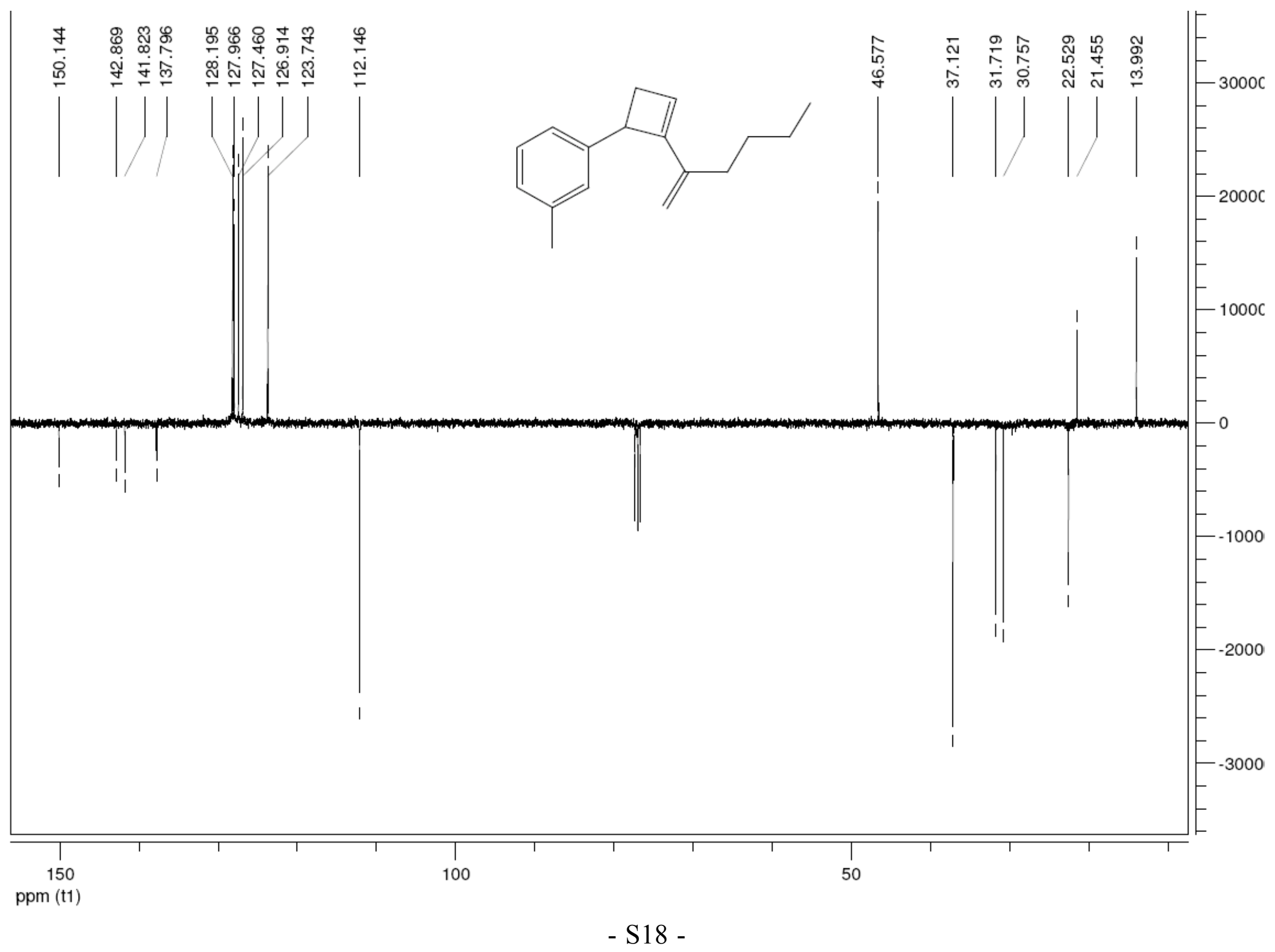




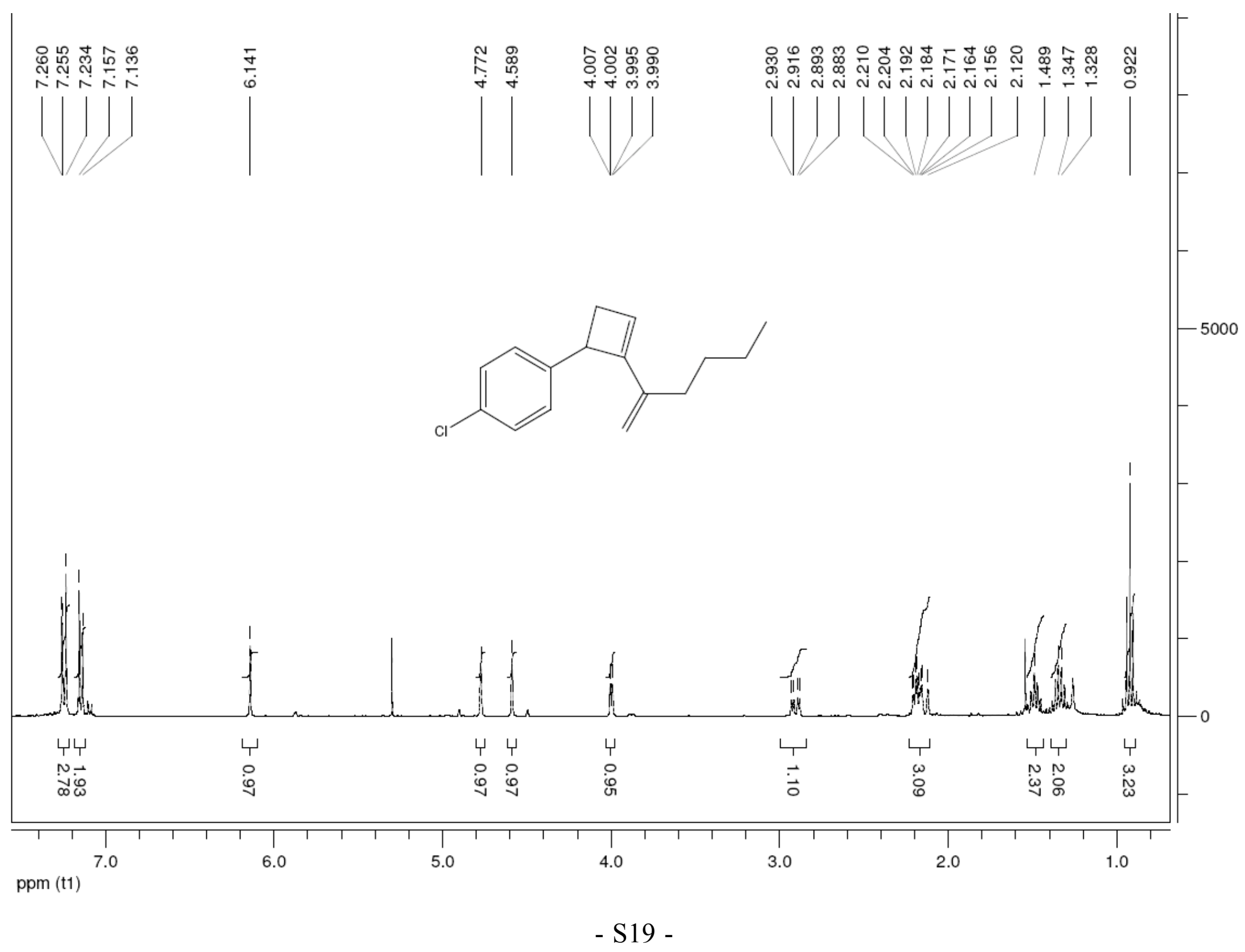




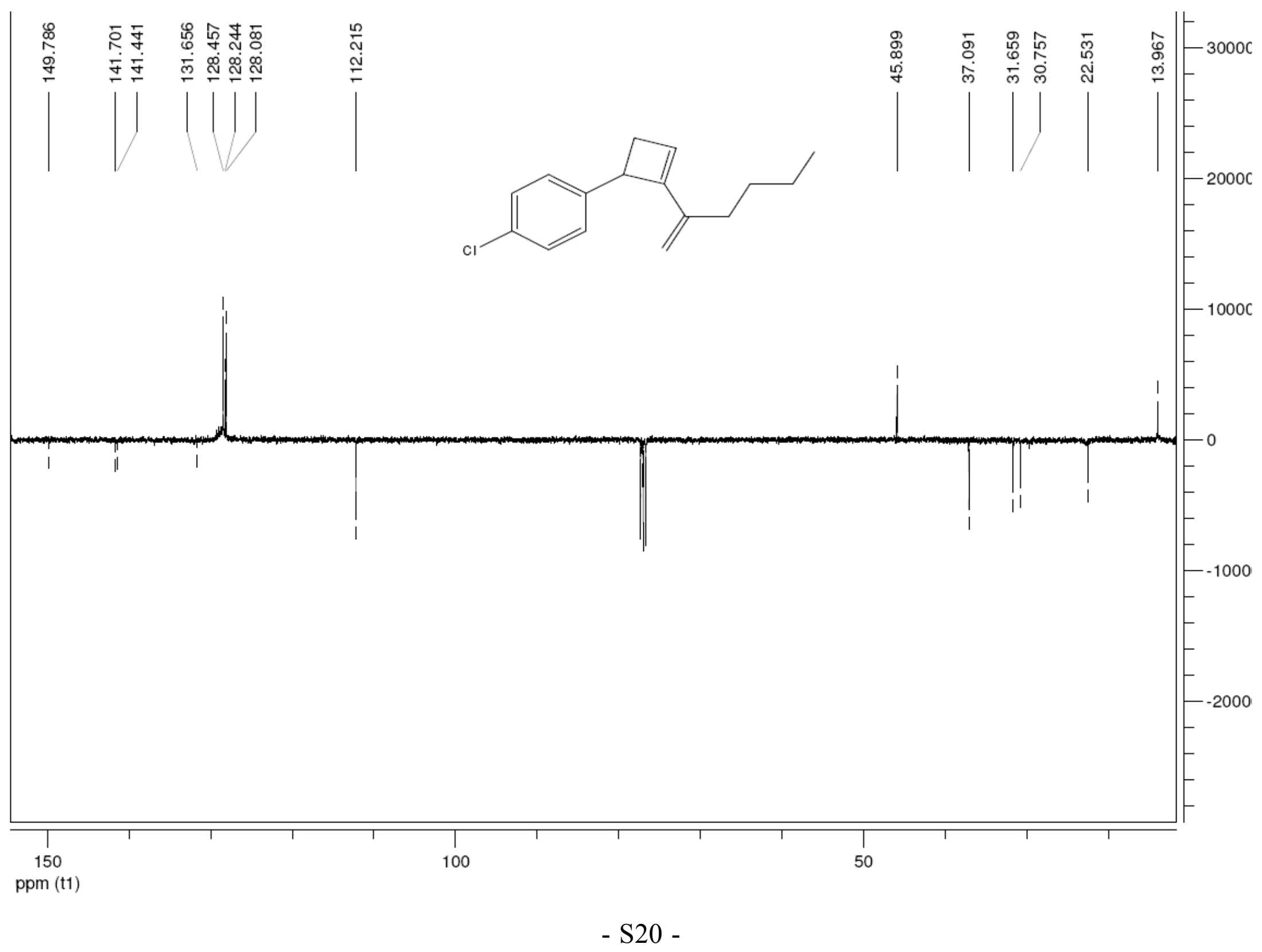




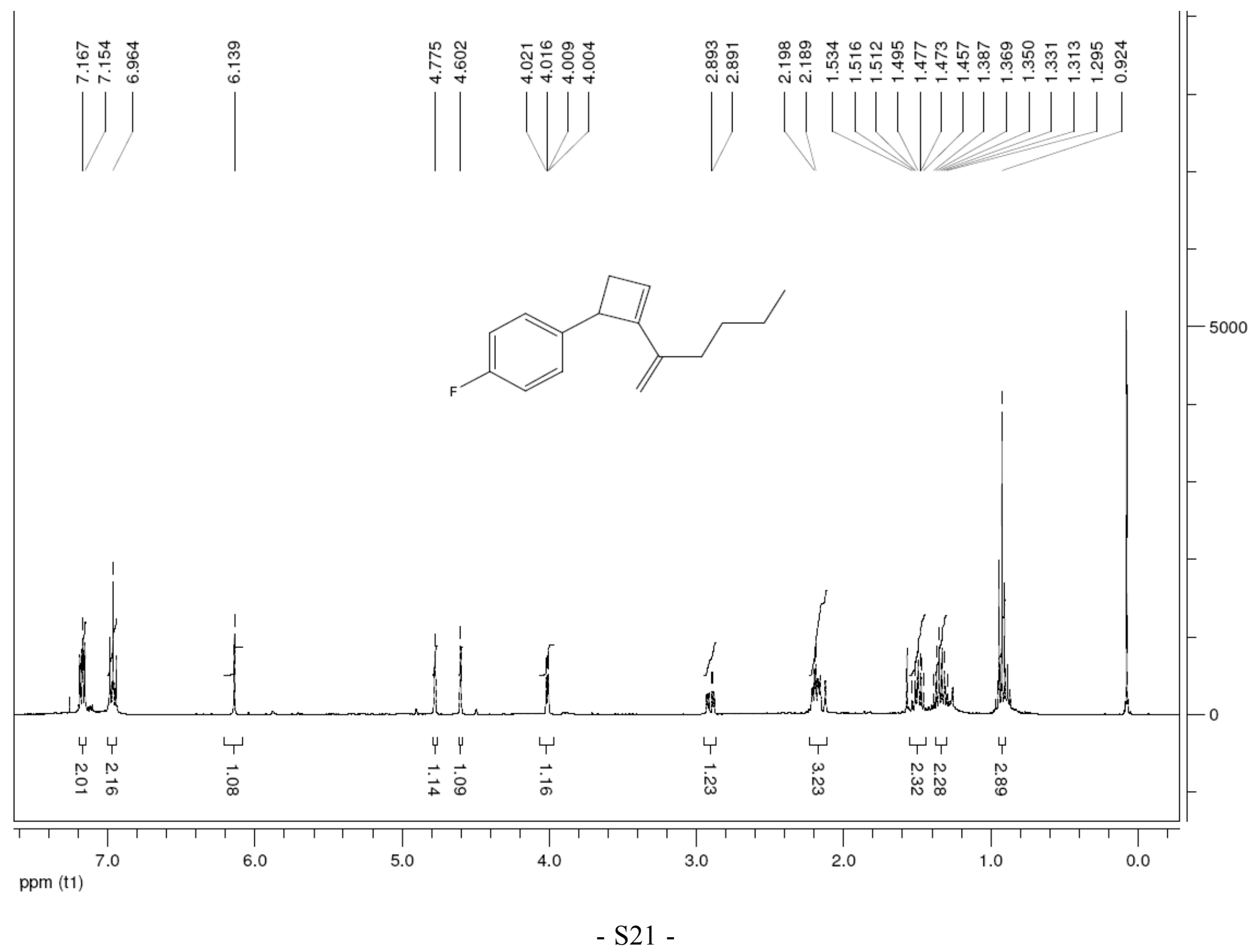




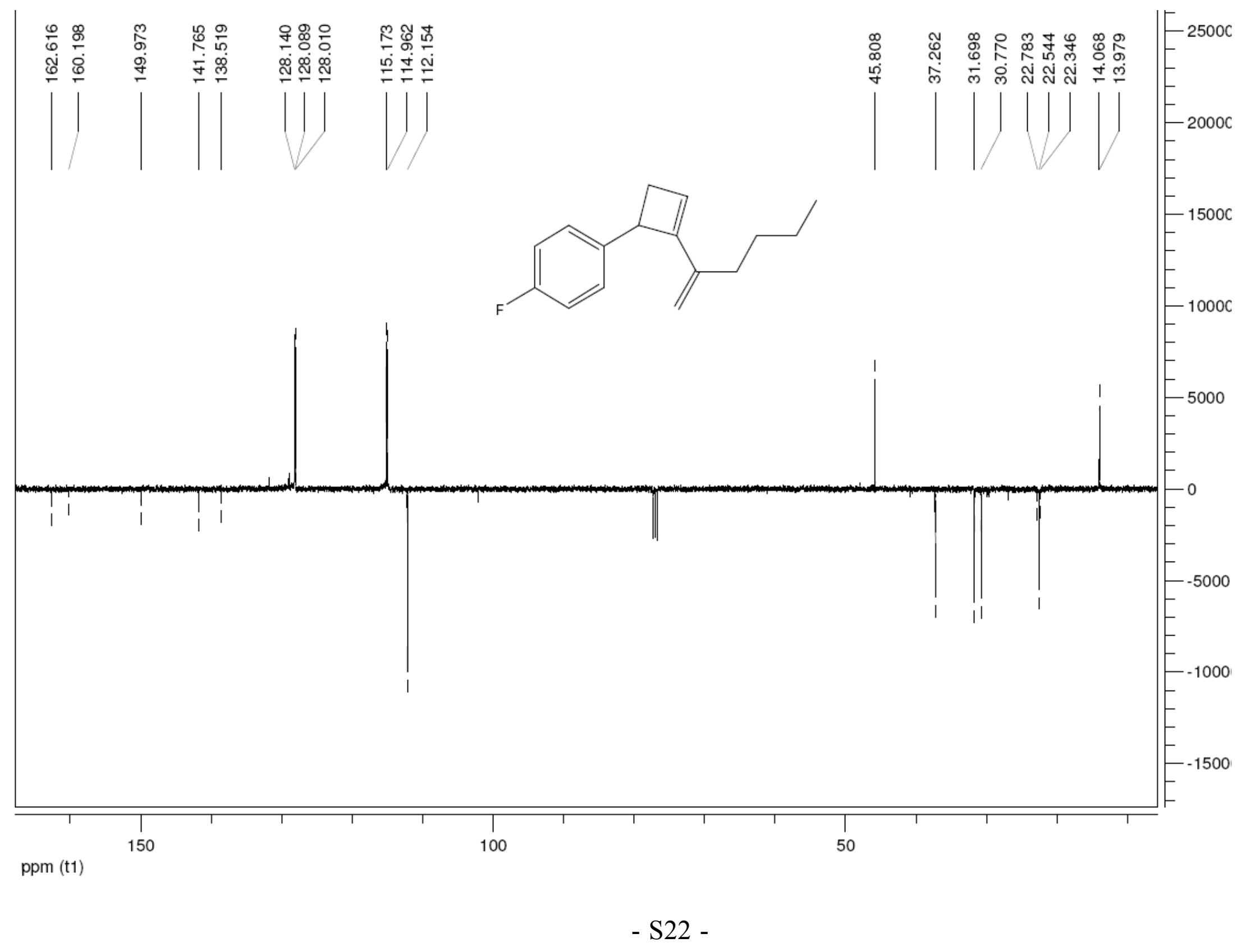




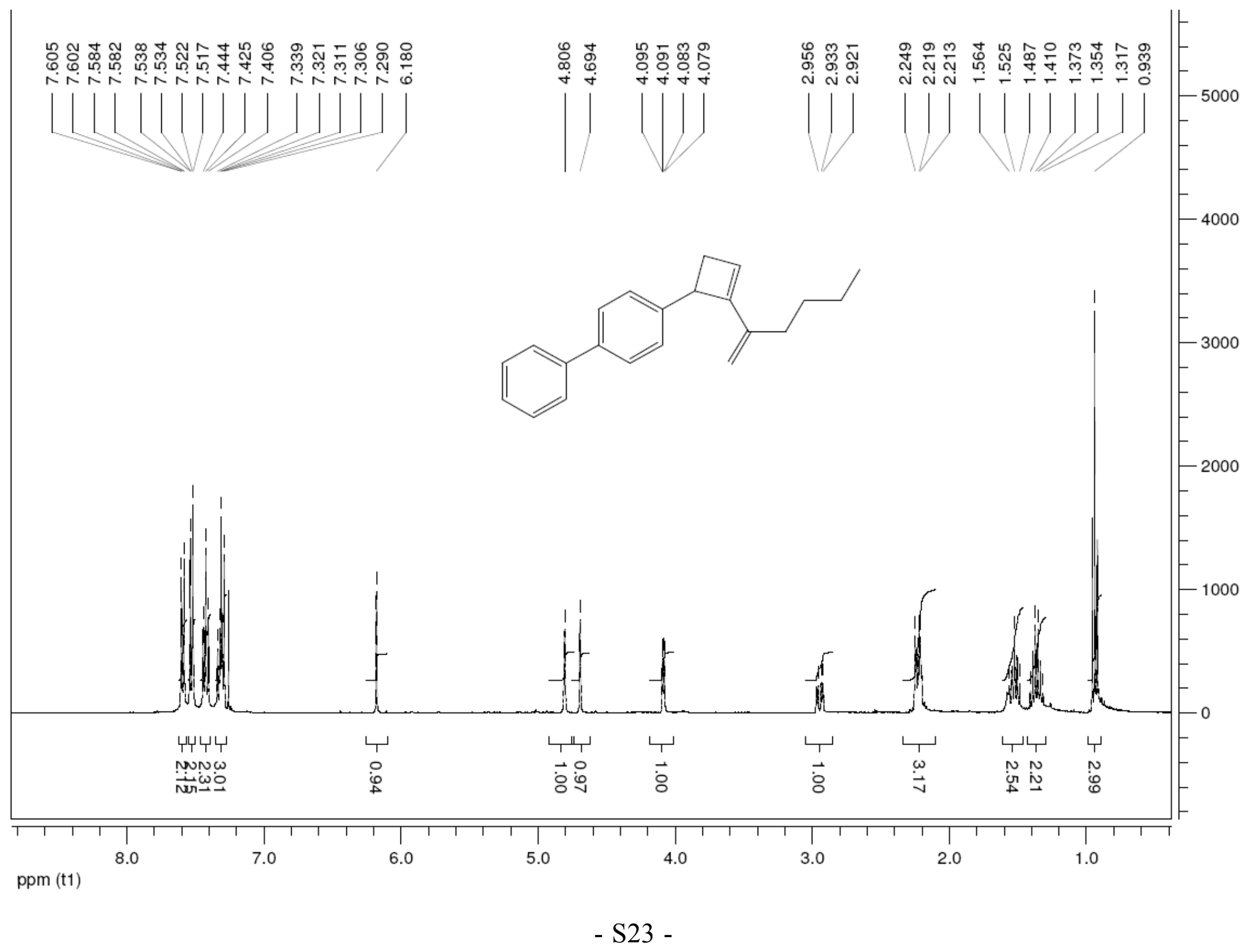




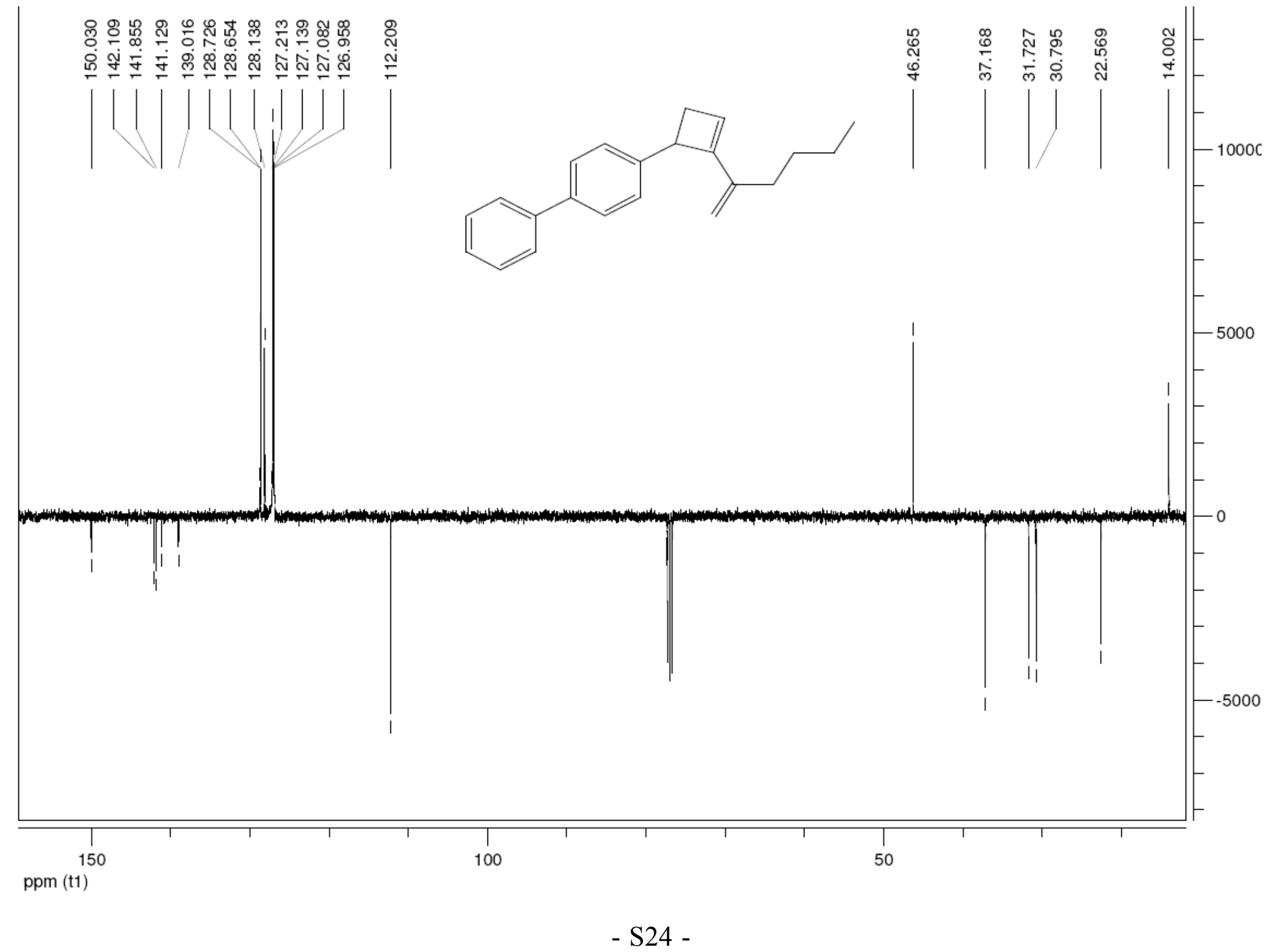




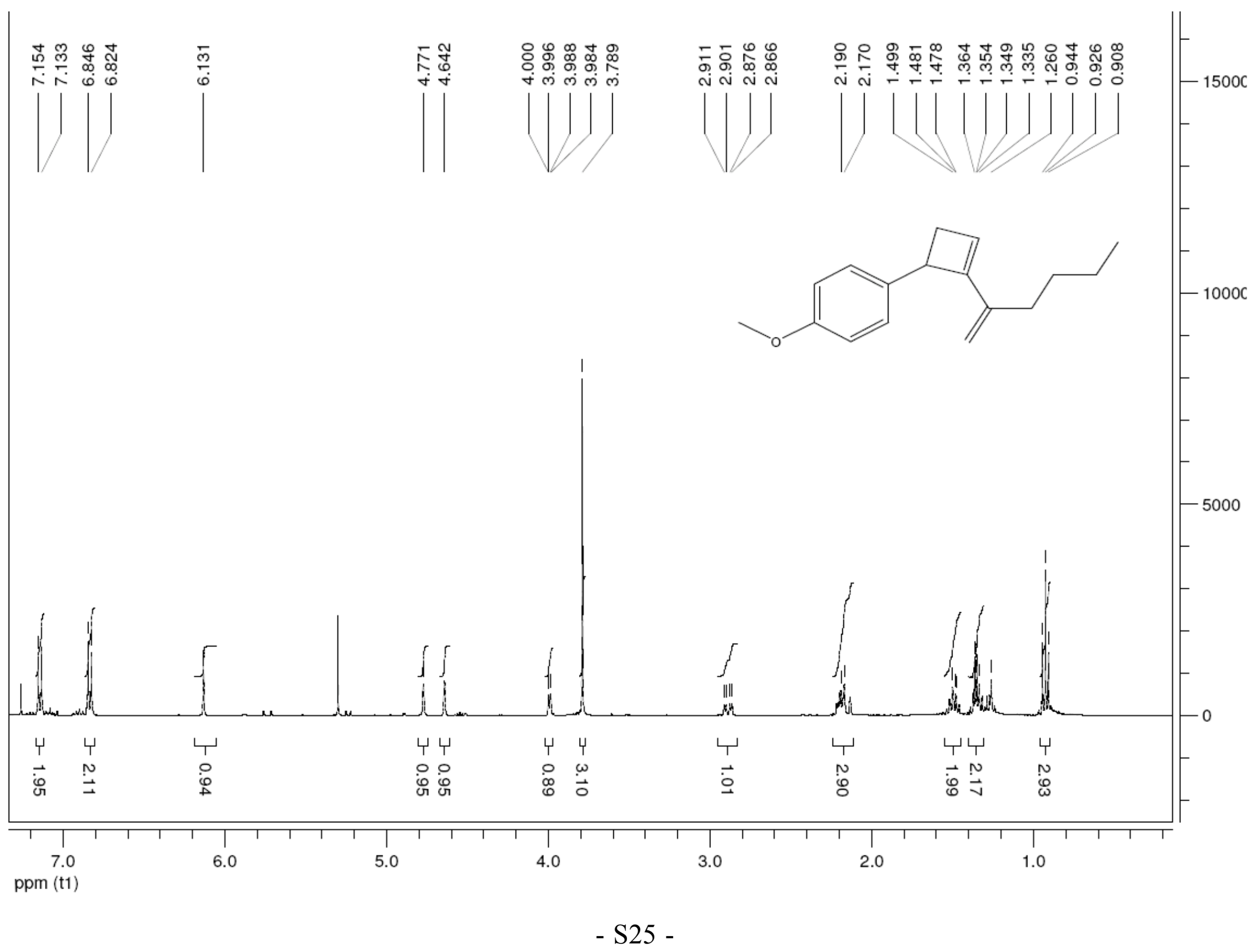




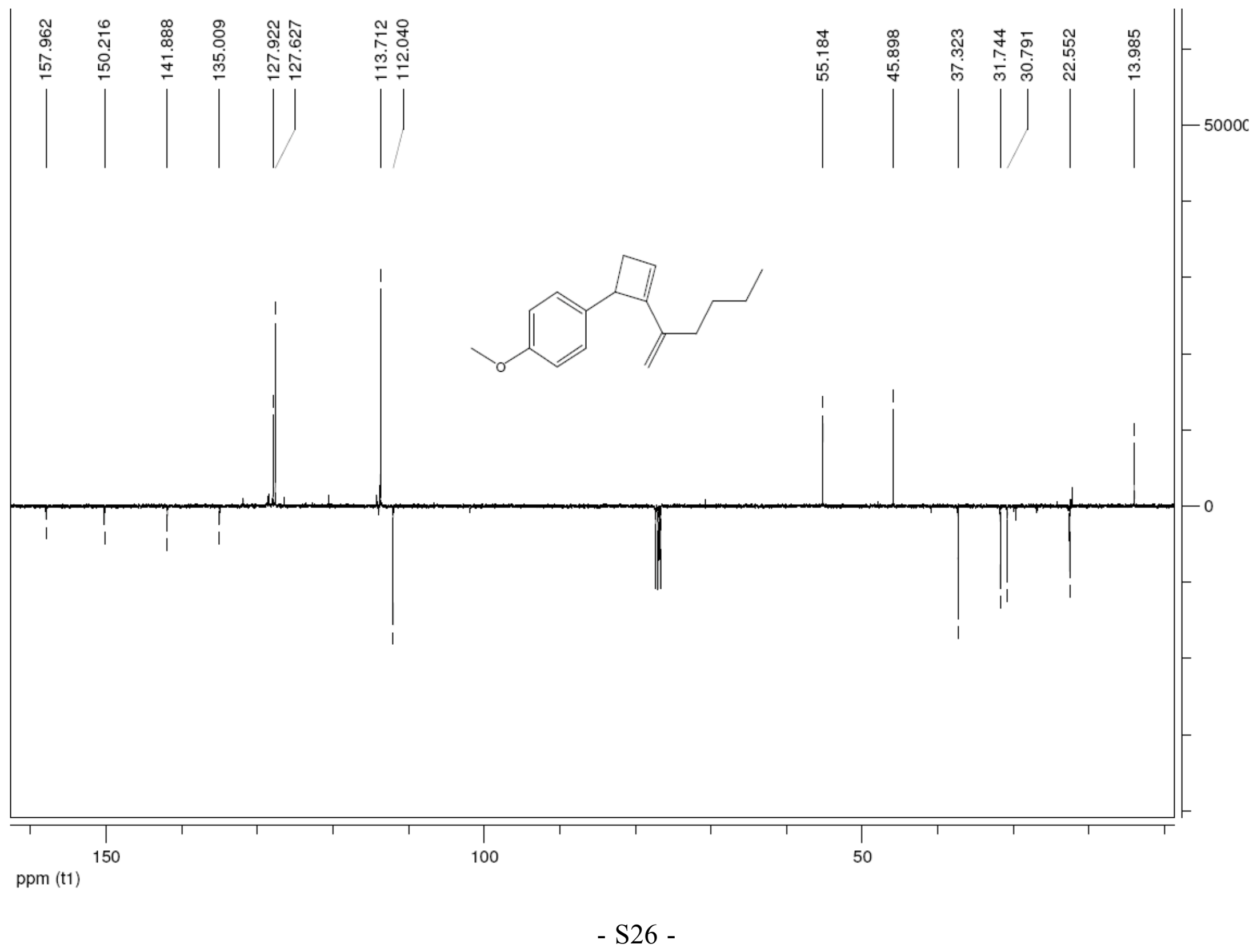




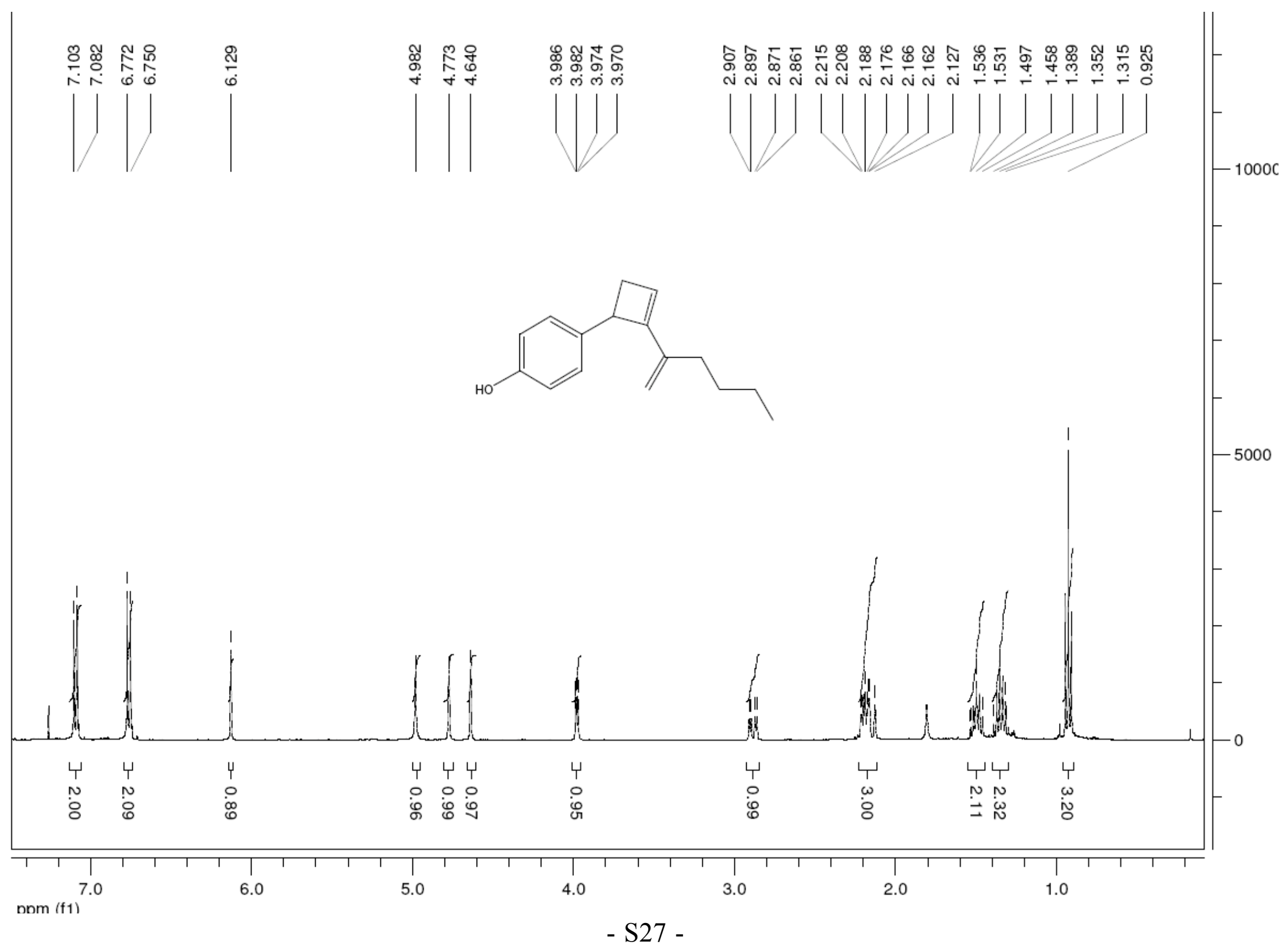




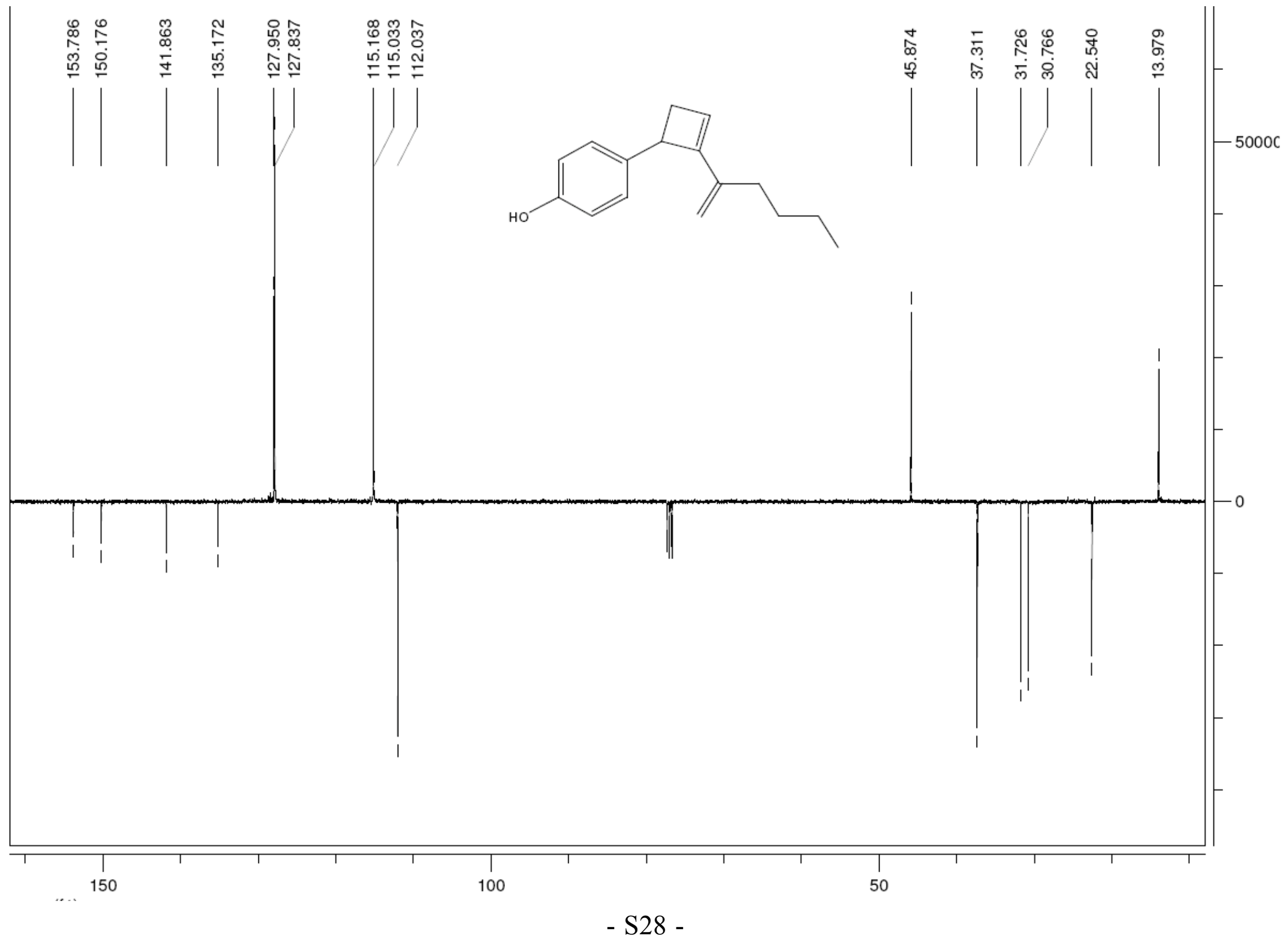




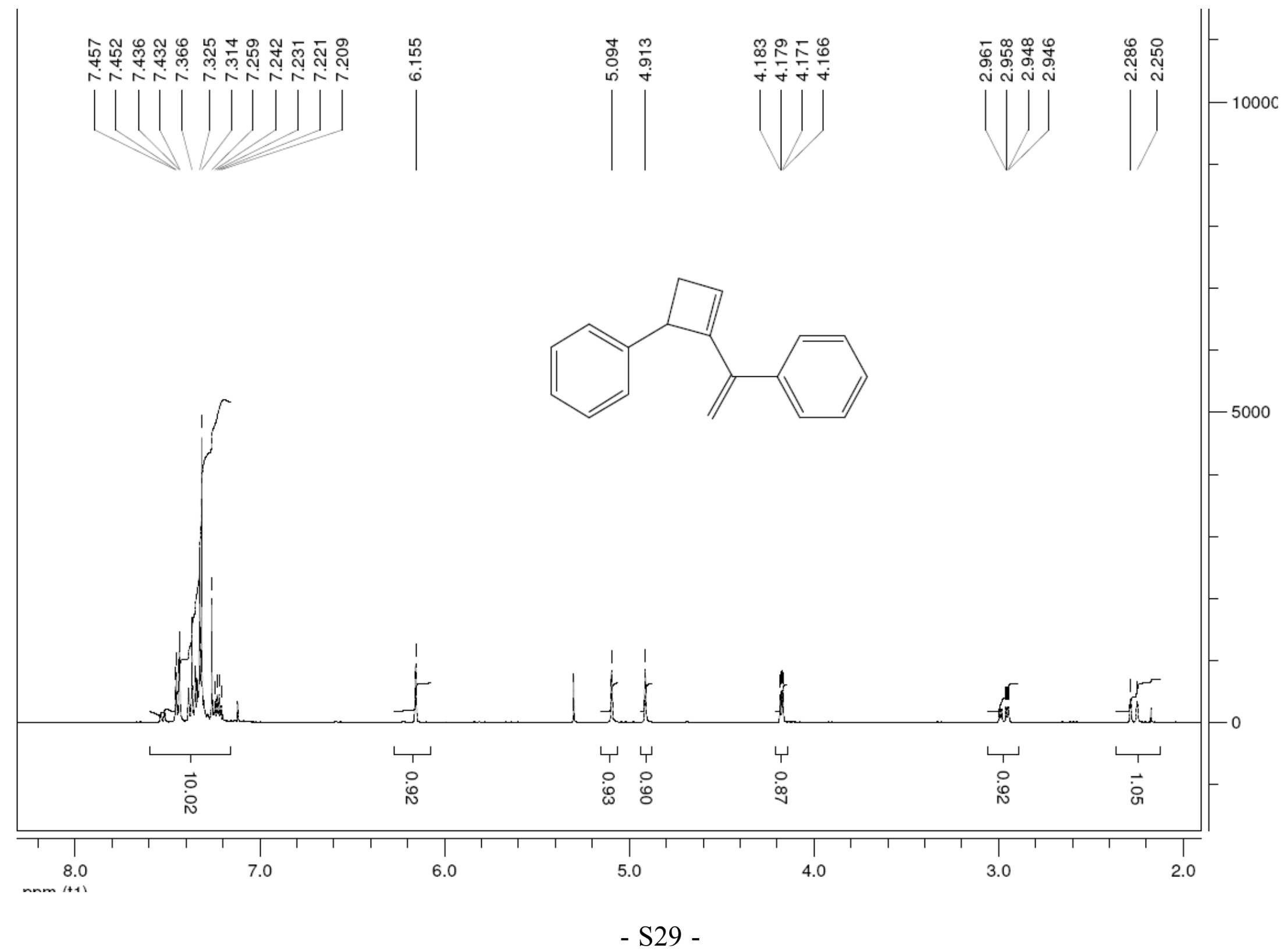




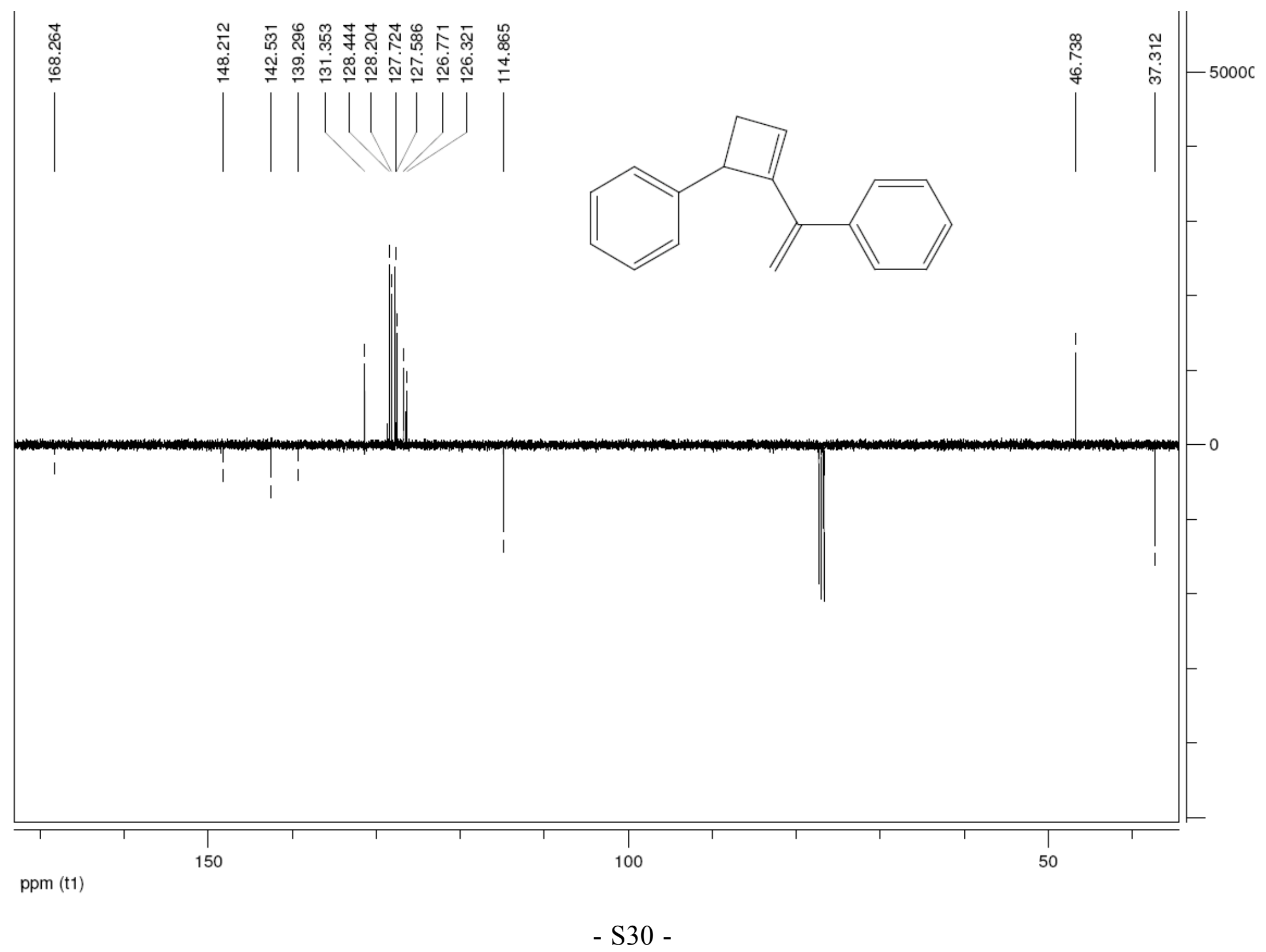




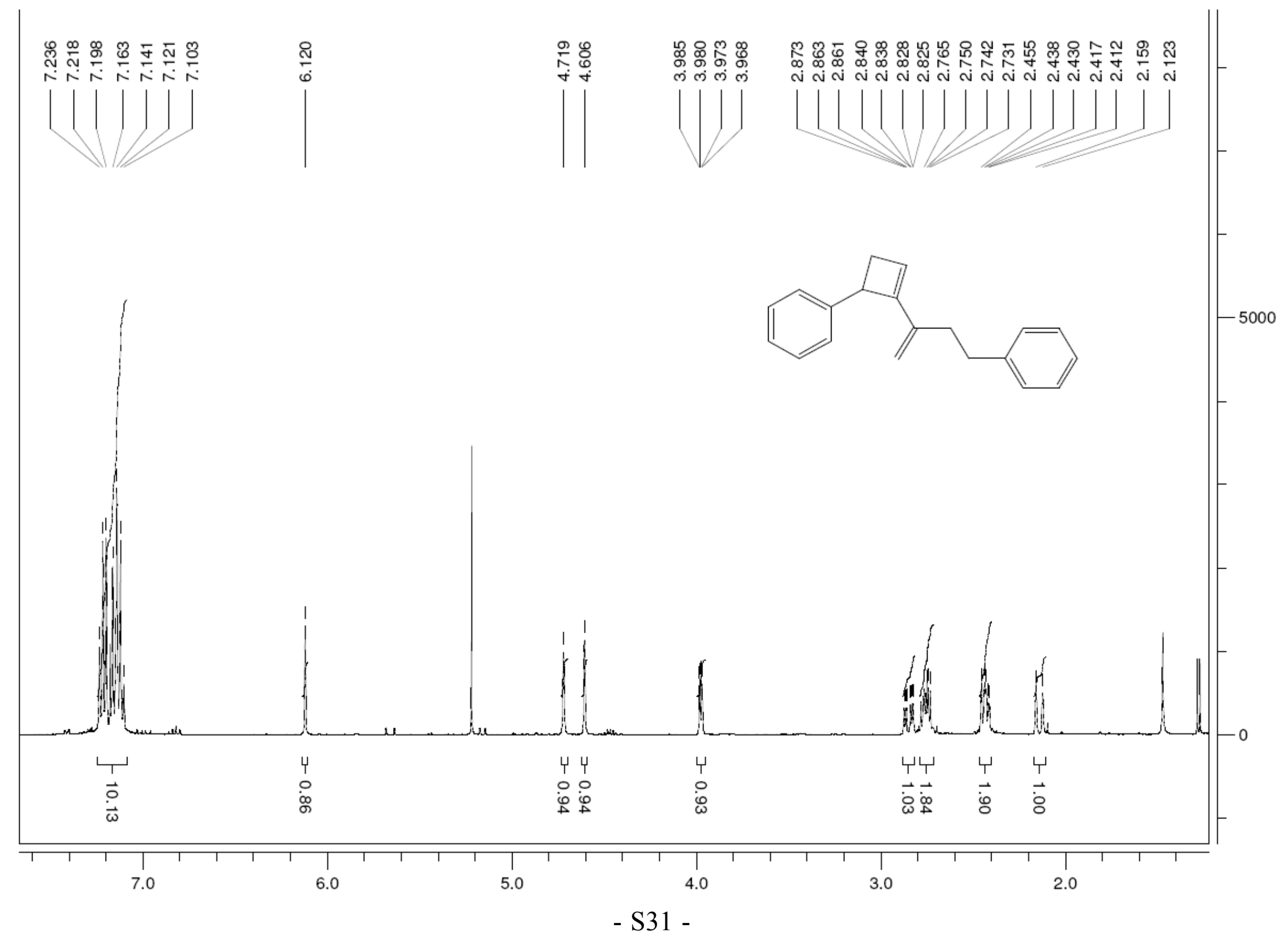




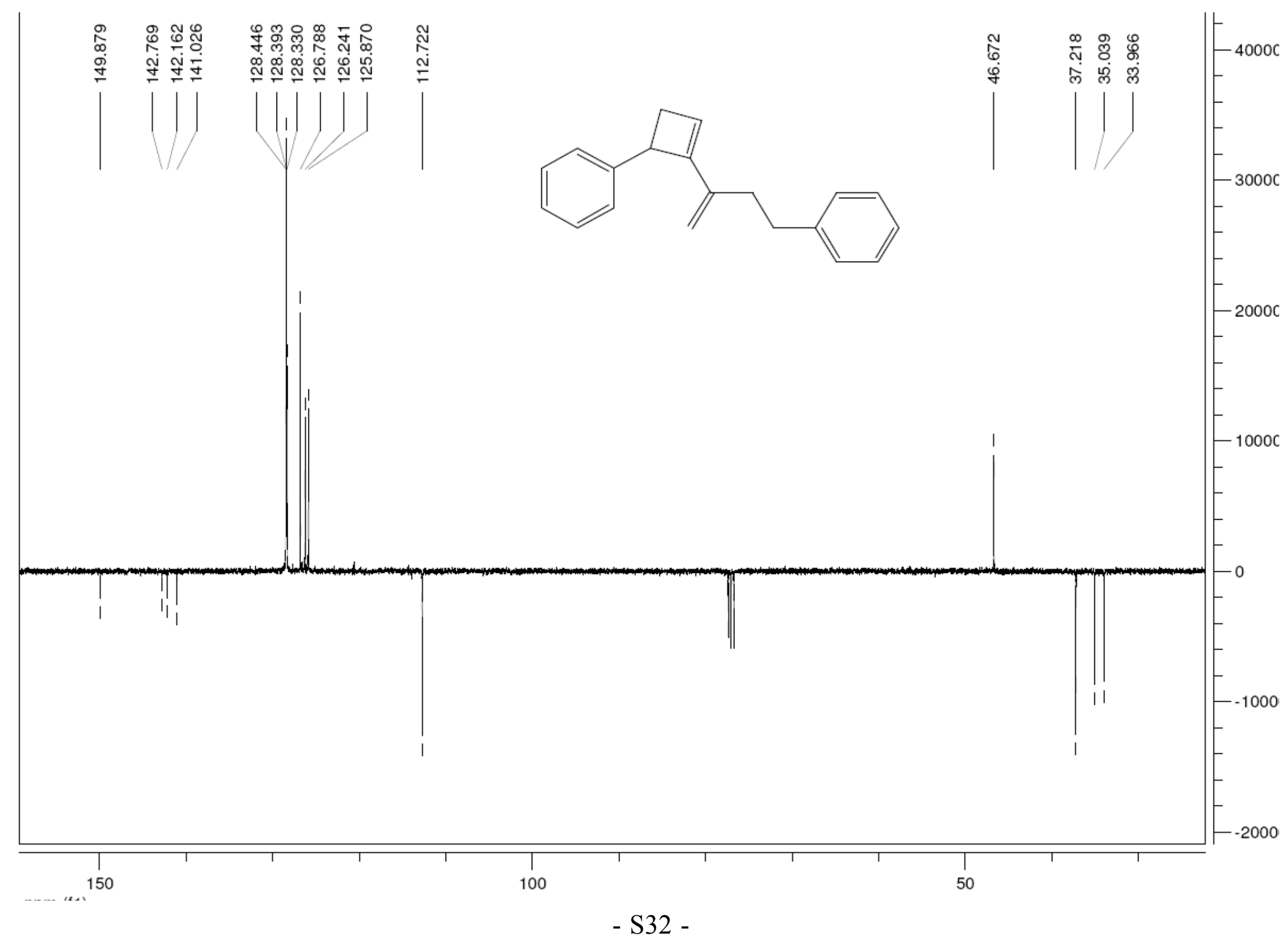




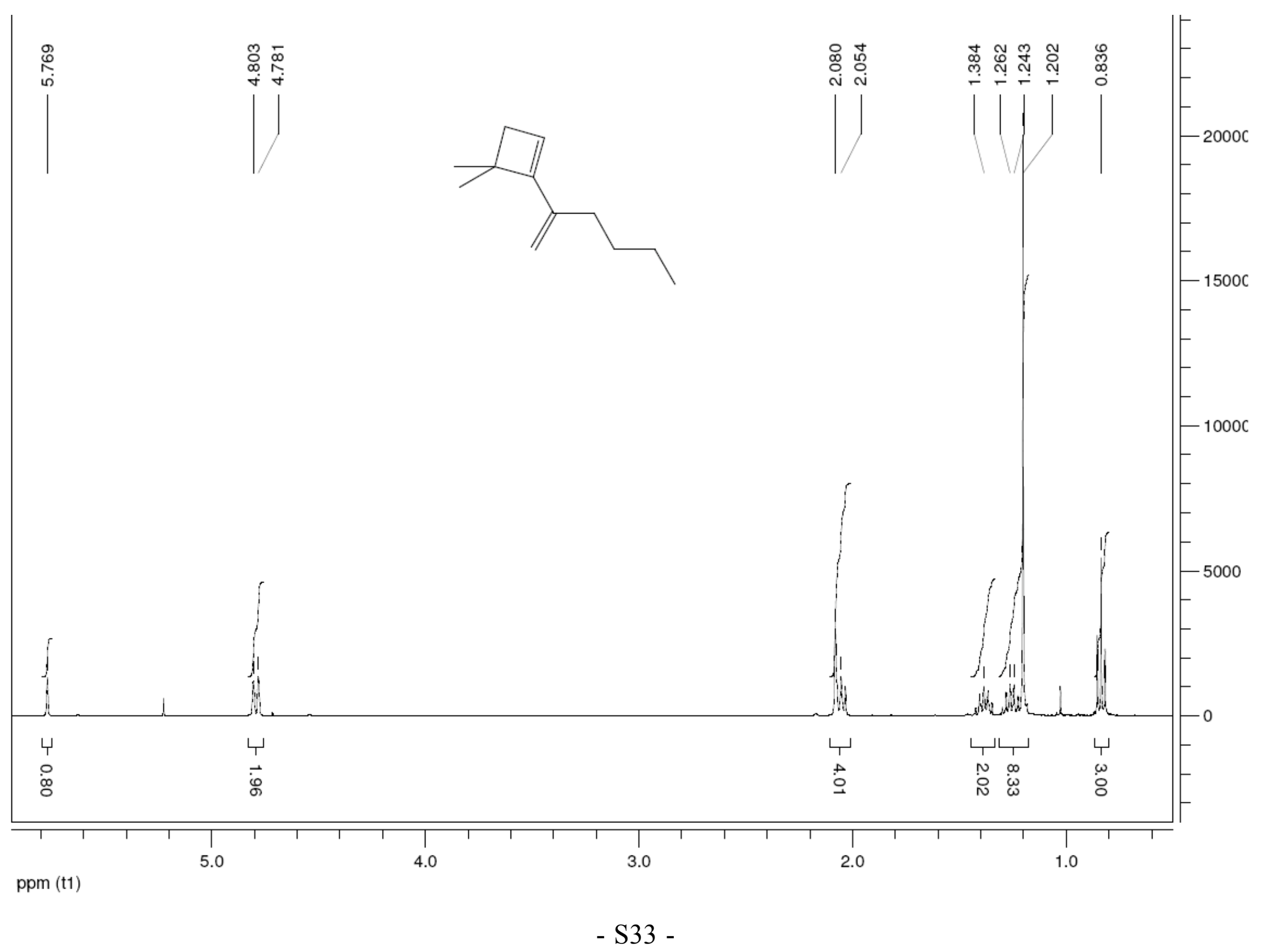




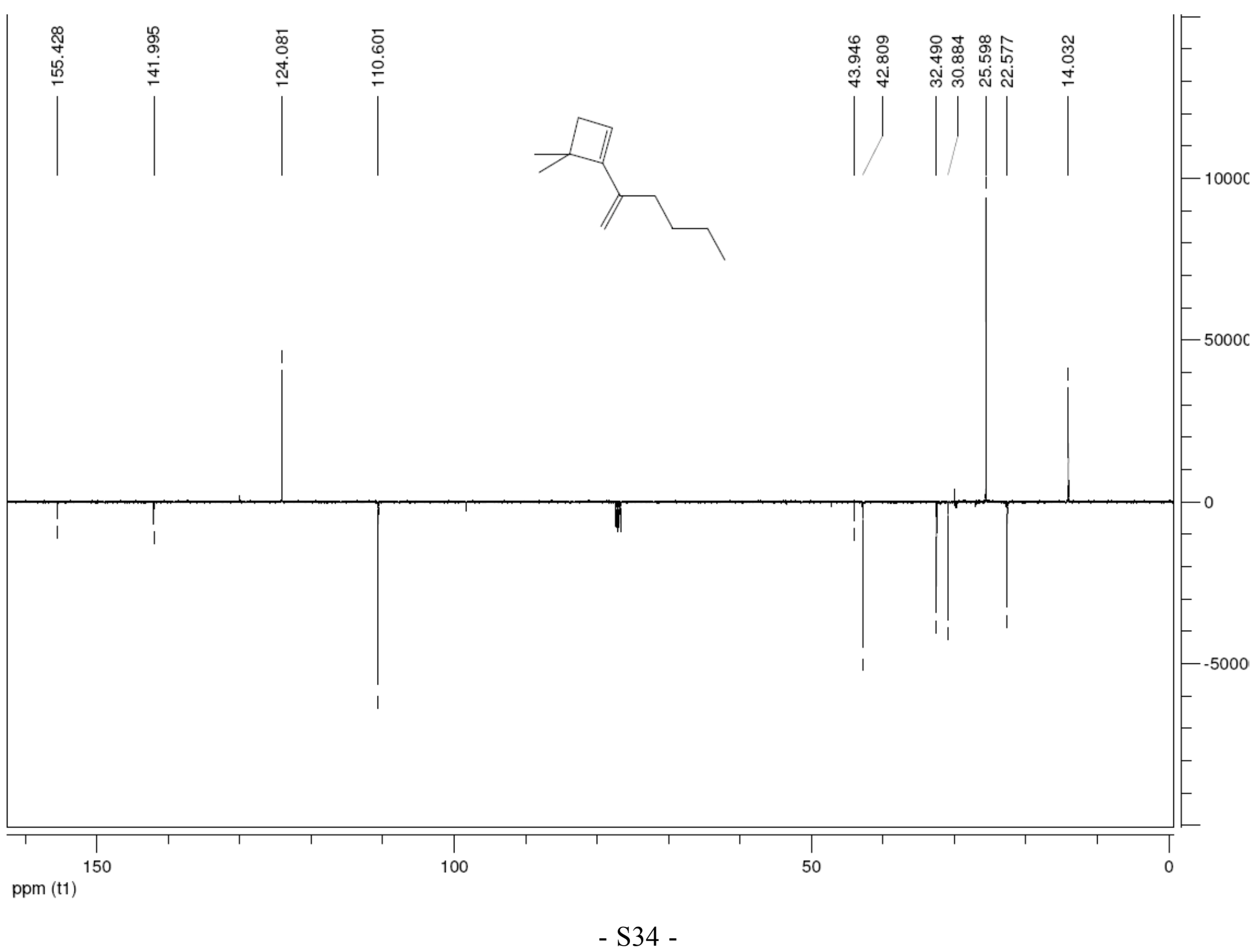




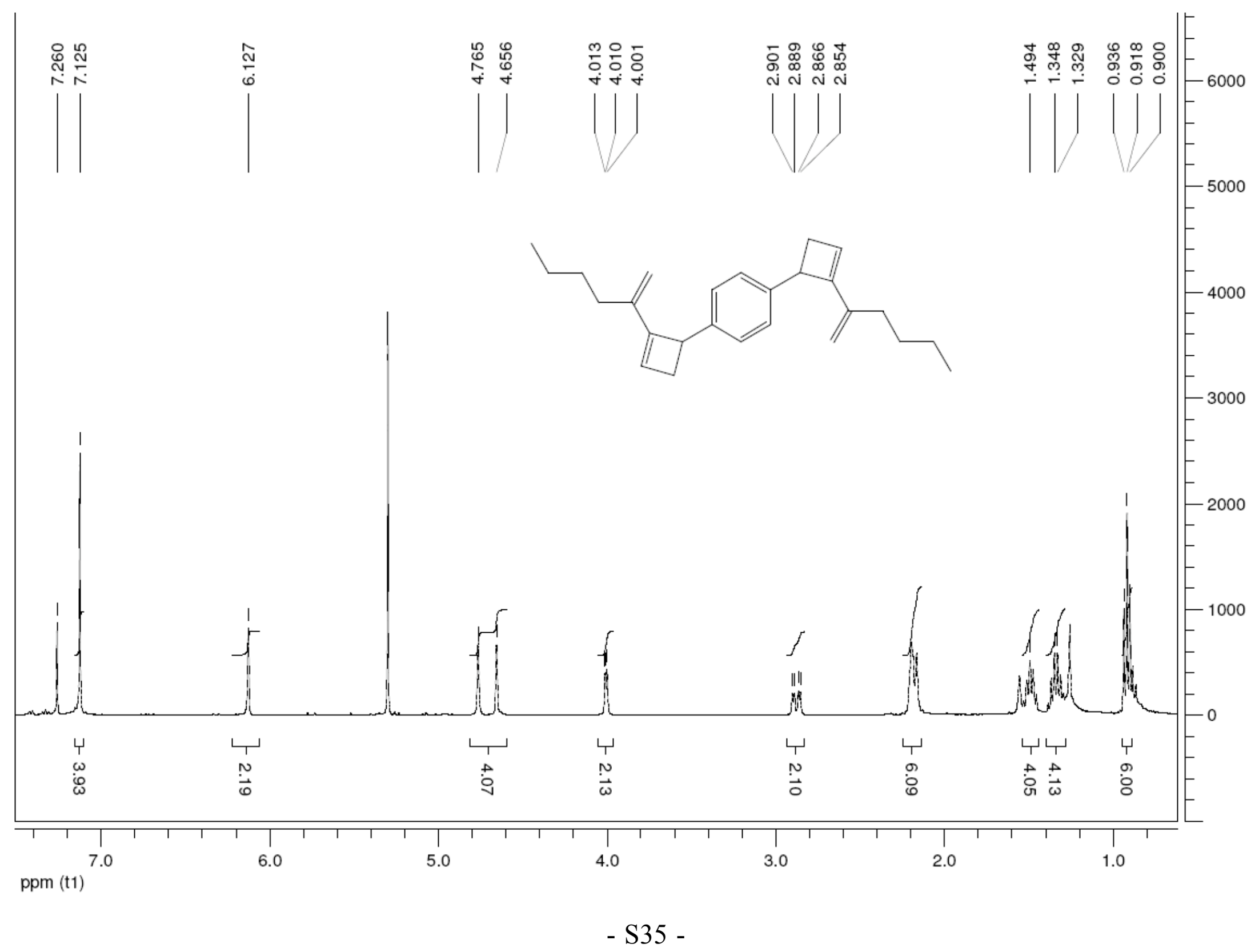




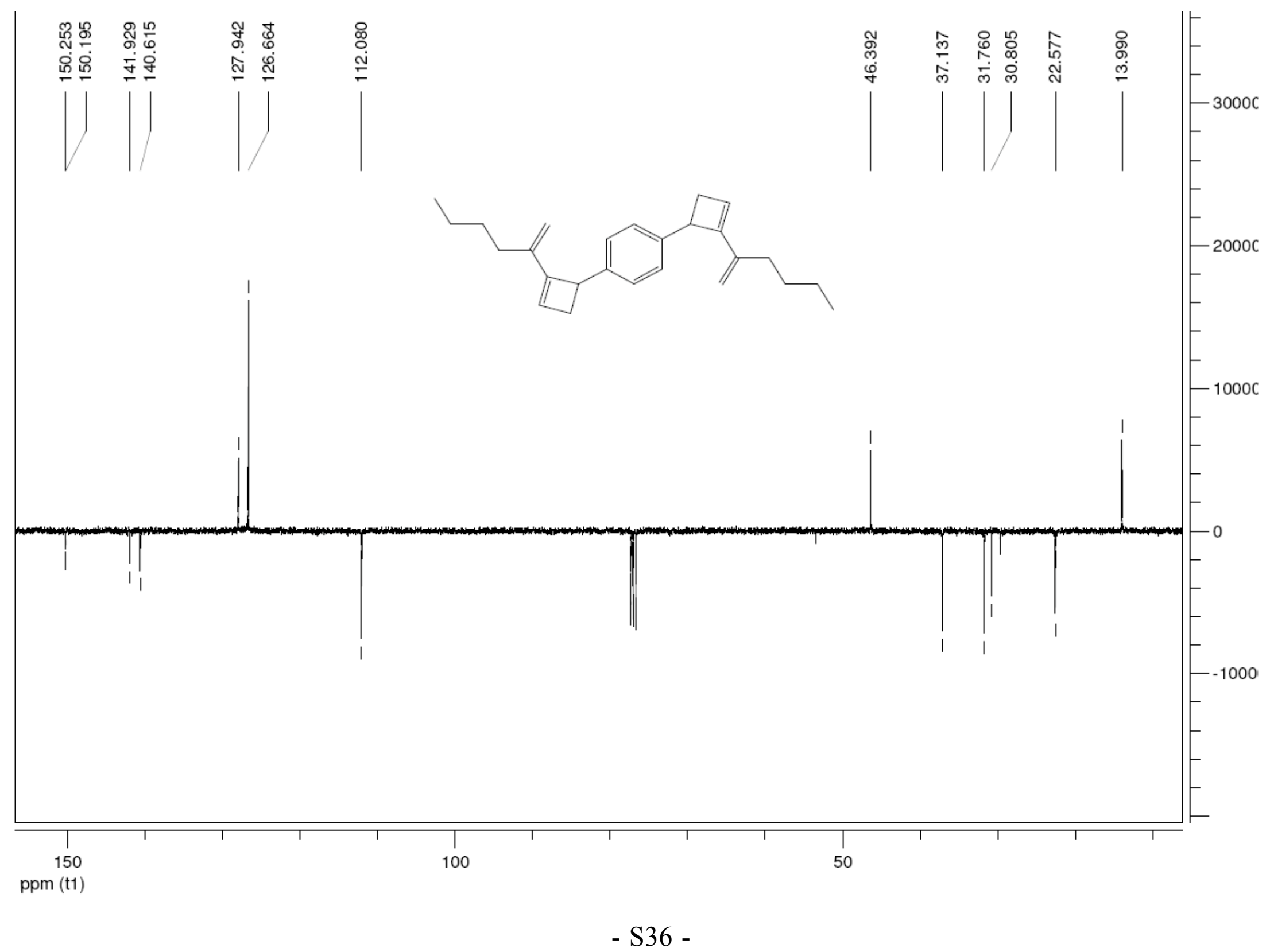




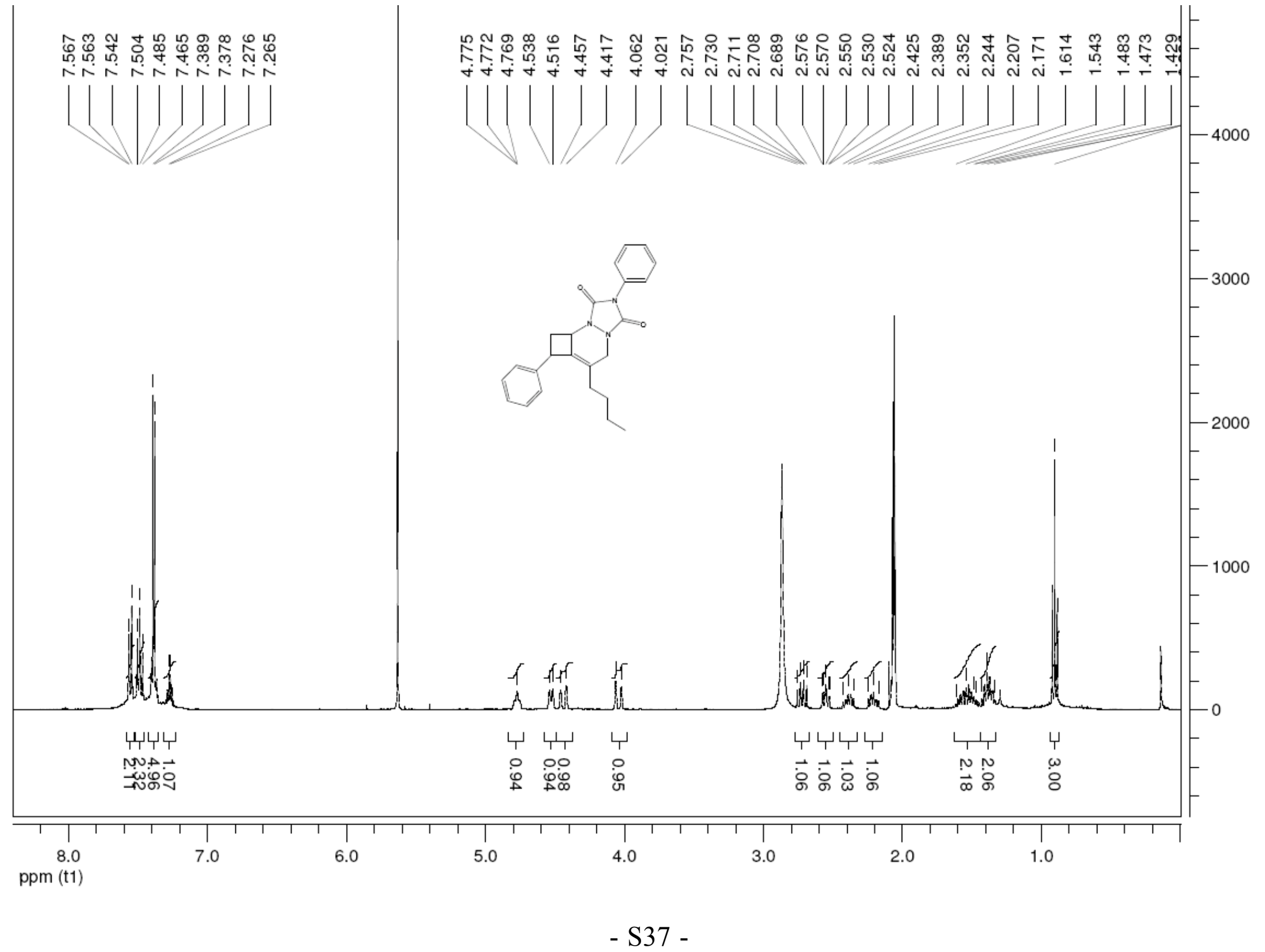




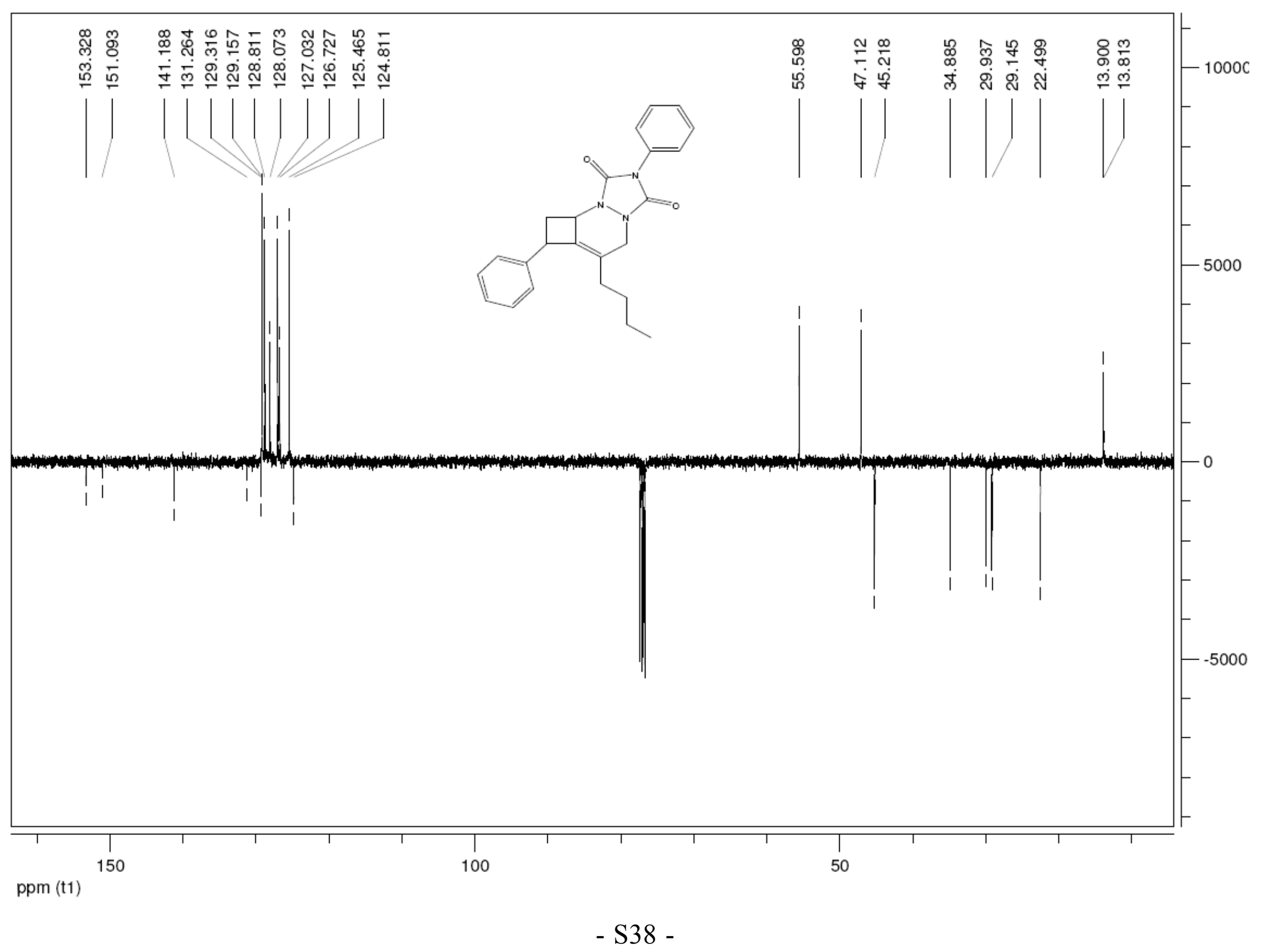

